\title{
Transition of blow-up mechanisms in $k$ equivariant harmonic map heat flow
}

\section{Pawe Biernat, Yukihiro Seki}

\begin{tabular}{|c|l|}
\hline Citation & OCAMI Preprint Series \\
\hline Issue Date & 2019 \\
\hline Type & Preprint \\
\hline Textversion & Author \\
\hline Relation & $\begin{array}{l}\text { The following article has been submitted to Nonlinearity. } \\
\text { This is not the published version. Please cite only the published version. The } \\
\text { article has been published in final form at } \\
\text { https://doi.org/10.1088/1361-6544/ab74f4 }\end{array}$ \\
\hline Is version of & $\underline{\text { https://doi.org/10.1088/1361-6544/ab74f4 } .}$ \\
\hline
\end{tabular}

From: Osaka City University Advanced Mathematical Institute http://www.sci.osaka-cu.ac.jp/OCAMI/publication/preprint/preprint.html 


\title{
Transition of blow-up mechanisms in $k$-equivariant harmonic map heat flow
}

\author{
Paweł Biernat ${ }^{*} \quad$ Yukihiro Seki ${ }^{\dagger}$
}

\begin{abstract}
In the present article, we consider blow-up phenomena appearing in $k$-equivariant harmonic map heat flow from $\mathbb{R}^{d}$ to a unit sphere $\mathbb{S}^{d} \subset \mathbb{R}^{d+1}$ :

$$
u_{t}=u_{r r}+\frac{d-1}{r} u_{r}-\frac{k(d+k-2)}{2 r^{2}} \sin (2 u), \quad r>0, t>0 .
$$

Here the scalar variable $u$ stands for latitudinal angle on $\mathbb{S}^{d}$ from the north pole $(u=0)$ to the south pole $(u=\pi)$. The integer $k \geq 1$ corresponds to the eigenvalues associated to eigenmaps $\Omega_{k}: \mathbb{S}^{d-1} \rightarrow \mathbb{S}^{d-1}$, that is, harmonic maps with constant energy density. We prove constructively the existence of asymptotically non-self-similar blow-up solutions with precise description of their local space-time profiles. The blow-up solutions arise from, depending on the combination of $d$ and $k$, two different approximations of the nonlinear term: either through a Dirac mass supported at the origin or via a Taylor expansion around equator map $u=\pi / 2$. Transition of the blow-up mechanisms arises, accordingly.
\end{abstract}

Key words: non-self-similar; harmonic map flow; matched asymptotic expansions 2010 Mathematics subject classification: 35K91; 35B44; 35C20.

\section{Introduction}

As a continuation of our previous work [8], we study the singularity formation of harmonic map heat flow from $\mathbb{R}^{d}$ to $\mathbb{S}^{d} \subset \mathbb{R}^{d+1}$, i.e., the equation for map $F: \mathbb{R}^{d} \times \mathbb{R}_{+} \rightarrow \mathbb{S}^{d}$ :

$$
\partial_{t} F=\Delta F+|\nabla F|^{2} F
$$

a gradient flow for the Dirichlet energy

$$
E[F]=\frac{1}{2} \int_{\mathbb{R}^{d}}|\nabla F|^{2} d x .
$$

Let $\Omega_{k}: \mathbb{S}^{d-1} \rightarrow \mathbb{S}^{d-1}$ denote an eigenmap, i.e., a harmonic map with constant energy density $\left|\nabla \Omega_{k}\right|^{2}=\Lambda_{k}$. From the general theory (cf. [11, Chapter VIII]), we know that

$$
\Lambda_{k}=k(d+k-2)
$$

*LIMES Institute, University of Bonn. Carl-Troll-Str. 31, 53115 Bonn, GERMANY

${ }^{\dagger}$ Graduate school of Mathematical Sciences, University of Tokyo, Corresponding to: yseki@ms.u-tokyo.ac.jp , 3-8-1 Komaba Meguro-ku Tokyo 153-8914, JAPAN. 
and $\Omega_{k}$ is a $d$-tuple consisting of eigenfunctions of $-\Delta_{\mathbb{S}^{d-1}}$ and represented by homogeneous polynomials of order $k$ subject to a condition $|\Omega|^{2}=1$. We particularize our analysis here to, so called, $k$-equivariant map:

$$
F(x, t)=\left(\Omega_{k}\left(\frac{x}{r}\right) \sin u(r, t), \cos u(r, t)\right),
$$

where $r=|x|, x \in \mathbb{R}^{d}$. The scalar variable $u$, on which we assume radial symmetricity with respect to $x$, stands for latitudinal angle on $\mathbb{S}^{d}$ from the north pole $(u=0)$ to the south pole $(u=\pi)$. Direct computations then show that

$$
\begin{aligned}
\partial_{r} F & =\partial_{r} u\left(\Omega_{k} \cos u,-\sin u\right), \\
\partial_{r r} F & =\partial_{r r} u\left(\Omega_{k} \cos u,-\sin u\right)-\left(\partial_{r} u\right)^{2}\left(\Omega_{k} \sin u, \cos u\right), \\
\nabla_{\mathbb{S}^{d-1}} F & =\left(\left(\nabla_{\mathbb{S}^{d-1}} \Omega_{k}\right) \sin u, 0\right), \quad \Delta_{\mathbb{S}^{d-1}} F=\left(\left(\Delta_{\mathbb{S}^{d-1}} \Omega_{k}\right) \sin u, 0\right), \\
\partial_{t} F & =\partial_{t} u\left(\Omega_{k} \cos u,-\sin u\right) .
\end{aligned}
$$

The energy density is expressed as

$$
|\nabla F|^{2}=\left|\partial_{r} F\right|^{2}+\frac{1}{r^{2}}\left|\nabla_{\mathbb{S}^{d-1}} F\right|^{2}=\left(\partial_{r} u\right)^{2}+\frac{\Lambda_{k}}{r^{2}} \sin ^{2} u .
$$

Using the decomposition of the Laplacian into its radial and transversal parts, we get

$$
\begin{aligned}
\Delta F & =\partial_{r r} F+\frac{d-1}{r} \partial_{r} F+\frac{1}{r^{2}} \Delta_{\mathbb{S}^{d-1}} F \\
& =\left(\partial_{r r} u+\frac{d-1}{r} \partial_{r} u+\frac{\Lambda_{k}}{r^{2}}\right)\left(\Omega_{k} \cos u,-\sin u\right)-\left(\partial_{r} u\right)^{2}\left(\Omega_{k} \sin u, \cos u\right) .
\end{aligned}
$$

Consequently, the harmonic map heat flow (1.1) is reduced to a $k$-equivariant ansatz:

$$
\begin{aligned}
u_{t} & =u_{r r}+\frac{d-1}{r} u_{r}-\frac{k(d+k-2)}{2 r^{2}} \sin (2 u), \quad r>0, t>0, \\
u(r, 0) & =u_{0}(r), \quad r \geq 0 .
\end{aligned}
$$

Due to (1.5), the Dirichlet energy $E[F]$ can be written as $E[F]=\operatorname{Vol}\left(\mathbb{S}^{d-1}\right) E_{k}(u)$ with

$$
E_{k}(u)=\frac{1}{2} \int_{0}^{\infty}\left(\left(\partial_{r} u\right)^{2}+k(d+k-2) \frac{\sin ^{2} u}{r^{2}}\right) r^{d-1} d r
$$

The continuity at $r=0$ of the map $F(\cdot, t)$ as in (1.4) imposes boundary condition

$$
u(0, t)=0 .
$$

As a matter of fact, equation (1.6a) is a gradient flow for $E_{k}(u)$. As for the initial data, we assume that $u_{0}$ is a nonnegative function such that $u_{0}(r)=O\left(r^{k}\right)$ as $r \rightarrow 0$. Existence and uniqueness of local-in-time classical solution of (1.6a)-(1.6c) is shown by rather standard arguments. See [8, Proposition 3.1] for the case $k=1$. The proof begins with changing unknown variable as $\Phi_{1}(r, t)=u(r, t) / r$ in order to transform equation (1.6a) to another equation with a bounded nonlinear term. The same argument works for general $k \geq 1$ 
if we start with the transformation $\Phi_{k}(r, t)=u(r, t) / r^{k}$. We say that a solution $u$ of (1.6a)-(1.6c) blows up in a finite time $T$ if $u$ is smooth in $\mathbb{R}_{+} \times(0, T)$ and

$$
\limsup _{t \nearrow T} \sup _{r>0} \frac{u(r, t)}{r^{k}}=+\infty
$$

A blow-up is said to be of Type I if there exists a constant $C>0$ such that

$$
\sup _{r>0} \frac{u(r, t)}{r^{k}} \leq \frac{C}{\sqrt{T-t}}, \quad t<T
$$

and of type II otherwise. Type II blow-up means that the singularity structure is not in accordance with the scaling property of (1.6a). Much effort has been recently paid to determining blow-up types for (1.6a) with $k=1$. Despite its apparent simplicity, equation (1.6a) admits various blow-up mechanisms depending on the combination of $k$ and $d$. Below we just review some of the known results.

In the two-dimensional case $d=2$, a generic blow-up pattern is of type II and is realized by a shrinking harmonic map with finite energy so that the blow-up may be viewed as "bubbling" process, where some portion of energy is trapped inside the singularity $[3,29,30,37]$. In dimensions $3 \leq d \leq 6$, there exist self-similar solutions, which exhibit type I blow-up [12]. The shrinking self-similar solutions, together with expanding ones, can be used to describe global (possibly nonunique) weak solutions to (1.6a) $[5,6,16]$. Moreover, the stability property of the shrinking self-similar solutions constructed in [6] has been shown in [7]. On existence and regularity of weak solutions for rough initial data of finite Dirichlet energy, readers are referred to [10] and the references cited therein. Uniqueness of weak solutions in a class that includes blow-up solutions has been studied in [15]. The blow-up solutions constructed in [8], however, do not satisfy a condition of the uniqueness result in [15].

In higher dimensions $7 \leq d$, type I blow-up cannot occur [9]. The proof given in [9] is by contradiction, and hence no information on actual blow-up rate or the asymptotic profiles is obtained there. We aim at constructing typical examples of type II blow-up solutions with quantitative informations about their blow-up rate and profiles. In our previous article [8], we have proven that there exist a countably many type II blow-up solutions with exact rates, justifying some of the formal constructions in [4]. A stability result of such blow-up solutions has been obtained in [17]. For further results, see the introduction in [8].

The key point of the arguments in [8] consists in the linearization of (1.6a), after introducing backward self-similar variables $(\Phi ; y, s)$, around the singular map $\pi / 2$. The operator associated to the linearization is formally written as

$$
A v=-\frac{1}{\rho} \frac{\partial}{\partial y}\left(\rho \frac{\partial v}{\partial y}\right)+\frac{k(k+d-2)}{y^{2}} v, \quad \rho=y^{d-1} e^{-y^{2} / 4} .
$$

It is realized as a symmetric operator in $L_{r, \rho}^{2}\left(\mathbb{R}^{d}\right)$ (cf. (2.4) for the definition). Let us just point out the following essential fact:

The linearized operator $A$ is lower semi-bounded in $L_{r, \rho}^{2}\left(\mathbb{R}^{d}\right)$

$$
\text { if } d>d^{*}(k):=2+(2+2 \sqrt{2}) k \text {. }
$$


See Proposition 2.1 below. The spectrum of Friedrichs' extension, still denoted by $A$, consists only of simple eigenvalues $\left\{\lambda_{n}\right\}_{n=0}^{\infty} \subset \mathbb{R}$ and $\lambda_{n}=n-\gamma / 2(n=0,1, \ldots)$, where $\gamma=\gamma(k, d)>0$ is the constant defined in (1.9) below. Each blow-up solution constructed in [8] is associated to a stable eigenvalue $\lambda_{\ell}>0$ (for the case $k=1$ ). However, for each $n \in \mathbb{N}$ there exist $k \geq 1$ and $d>d^{*}(k)$ such that $\lambda_{n}=0$. In the present article we construct blow-up solutions associated to the neutral eigenvalues for each $k \geq 1$.

In the general case $k \geq 1$, Gastel [14] proved the existence of self-similar blow-up solutions for $d<d^{*}(k)$. So far, all the results on type II singularity in the high-dimensional case were given only for the case $k=1$. In the case $k \geq 2$, there are several dimensions where neutral eigenvalues appear. Recall the asymptotic property of the stationary solution $U_{1}(r)$ satisfying $U_{1}(0)=0$ and $\lim _{r \rightarrow 0} U_{1}(r) / r^{k}=1[4,26]$ : If $d>d^{*}(k)$, then

$$
U_{1}(r)=\frac{\pi}{2}-h r^{-\gamma}+O\left(r^{-\gamma-\omega}\right)
$$

as $r \rightarrow \infty$, where $h$ is a positive constant and

$$
\gamma=\frac{d-2-\omega}{2} \quad \text { with } \quad \omega=\omega_{k, d}=\sqrt{(d-2)^{2}-4 k(d-2)-4 k^{2}}
$$

is the smaller root of quadratic equation $\gamma^{2}-(d-2) \gamma+k(d+k-2)=0$.

We are now in a position to state our main results. As a main novelty, we show that type II blow-up solutions associated to neutral eigenvalues actually exist and their blow-up mechanisms are quite different depending on whether $2 \gamma>\omega$ (i.e., $4 \gamma>d-2$ ) or $2 \gamma<\omega$ (i.e., $4 \gamma<d-2)$. The simplest case, $\lambda_{1}=0$, happens if and only if $(k, d, \gamma)=(1,7,2)$, and hence condition $4 \gamma>d-2$ is satisfied. Suppose next that $\lambda_{2}$ vanishes. This is true if and only if $(k, d, \gamma)=(2,12,4)$ or $(3,27,4)$. Notice that condition $4 \gamma>d-2$ holds for the former triplet, whereas condition $4 \gamma<d-2$ holds for the latter one. In general, the both situations occur in accordance with suitable choice of $k \geq 2$ and $d>d^{*}(k)$ when a higher eigenvalue $\lambda_{n_{0}}\left(n_{0} \geq 2\right)$ vanishes (cf. Remark 2.2 below).

Theorem 1.1. Assume that $d>d^{*}(k)=2+(2+2 \sqrt{2}) k$ holds. Suppose that $\gamma=2 n_{0}$ for some integer $n_{0} \geq 1$. Then there exists a solution $u$ of $(1.6 \mathrm{a})-(1.6 \mathrm{~b})$, with $E_{k}\left(u_{0}\right)<\infty$, that blows up in a finite time $T$ and that fulfills the following properties:

(i) (Exact blow-up rate) There exists a constant $\theta \in(0,1)$ such that the limit

$$
\lim _{t \rightarrow T} \frac{(T-t)^{k / 2}}{|\log (T-t)|^{k / \delta}} \frac{u(r, t)}{r^{k}} \text { with } \delta=\min \{2 \gamma, \omega\}
$$

exists and is positive. The convergence is uniform on the set $\{0<r \leq C \mid \log (T-$ $\left.t)\left.\right|^{-2 \theta / \delta} \sqrt{T-t}\right\}$ for every constant $C>0$.

(ii) (Estimates in a neighborhood of the inner layer) There exists a positive smooth function $\varepsilon(s)$ satisfying, if $2 \gamma>\omega$,

$$
\begin{aligned}
& \varepsilon(s)=K s^{-1 / \omega}\{1+o(1)\}, \\
& \dot{\varepsilon}(s)=-\frac{K}{\omega} s^{-1 / \omega-1}\{1+o(1)\}
\end{aligned}
$$


and if $2 \gamma<\omega$,

$$
\begin{aligned}
& \varepsilon(s)=\tilde{K} s^{-1 / 2 \gamma}\{1+o(1)\}, \\
& \dot{\varepsilon}(s)=-\frac{\tilde{K}}{2 \gamma} s^{-1 / 2 \gamma-1}\{1+o(1)\}
\end{aligned}
$$

as $s \rightarrow \infty$ for some constants $K, \tilde{K}>0$, and

$$
\left|u(r, t)-U_{1}\left(\frac{r}{\varepsilon(s) \sqrt{T-t}}\right)\right| \leq \varepsilon(s)^{2 \theta} \Psi\left(\frac{r}{\varepsilon(s) \sqrt{T-t}}\right)
$$

for $r \leq \varepsilon(s)^{\theta} \sqrt{T-t}, t<T$, where $s=|\log (T-t)|$ and $\Psi$ is a positive smooth function satisfying

$$
\Psi(\xi)= \begin{cases}O\left(\xi^{k}\right) & \text { as } \xi \rightarrow 0 \\ O\left(\xi^{-\gamma}\right) & \text { as } \xi \rightarrow \infty\end{cases}
$$

(iii) (Estimates in self-similar regions) For every $M>0$ there exists a constant $C_{M}>0$ such that

$$
\begin{array}{r}
\left|u(r, t)-\frac{\pi}{2}+D \varepsilon(s)^{\gamma}\left(\frac{r}{\sqrt{T-t}}\right)^{-\gamma} L_{n_{0}}^{(\omega / 2)}\left(\frac{r^{2}}{4(T-t)}\right)\right| \leq C_{M} \varepsilon(s)^{\gamma+2 \theta}(T-t)^{\gamma / 2} r^{-\gamma} \\
\text { for } \varepsilon(s)^{\theta} \sqrt{T-t} \leq r \leq M \sqrt{T-t}, t<T, \quad \text { (1.15) }
\end{array}
$$

where $D=h \mathcal{N}$ with $\mathcal{N}=\mathcal{N}\left(n_{0}, d\right)>0$ is a constant and $L_{n}^{(\nu)}(x)$ denotes the associated Laguerre polynomial of order $n$ :

$$
L_{n}^{(\nu)}(x)=\frac{x^{-\nu} e^{x}}{n !} \frac{d^{n}}{d x^{n}}\left(e^{-x} x^{n+\nu}\right),
$$

and where $\varepsilon(s)$ is as above.

(iv) (Number of intersections) There exist exactly $n_{0}$ simple zeros $\left\{r_{j}(t)\right\}_{j=1}^{n_{0}}$ of $u(\cdot, t)-$ $\pi / 2$ in $(0, \infty)$ for every $0 \leq t<T$, which satisfy $r_{j}(t)=O(\sqrt{T-t})$ as $t \nearrow T$ for $j=1, \ldots, n_{0}$.

Remark 1.2. It is readily seen that $\sup _{r>0} u(r, t) / r^{k}$ is attained in the region where $r \leq$ $C \varepsilon(s) \sqrt{T-t}, C>0$, for each $t \in(0, T)$ and not outside a ball with radius of order greater than $\varepsilon(s) \sqrt{T-t}$. In particular, there is a constant $M>0$ such that

$$
\sup \left\{\frac{u(r, t)}{r^{k}} ; \varepsilon(s)^{\theta} \sqrt{T-t} \leq r\right\} \leq \frac{M|\log (T-t)|^{k \theta / \delta}}{(T-t)^{k / 2}} .
$$

Remark 1.3. The constant $\pi / 2$ is a stationary solution of (1.6a) as well as of the rescaled equation (2.1c) below, although it does not satisfy the boundary condition (1.6c). The associated Laguerre polynomials in (1.15) appear in the expression of the eigenfunctions:

$$
\phi_{n_{0}}(y)=\mathcal{N} y^{-\gamma} L_{n_{0}}^{(\omega / 2)}\left(y^{2} / 4\right)
$$

for the linearized operator around $\pi / 2$ in the self-similar variables (cf. (2.8) below). The even integer assumption of $\gamma$ is merely for guaranteeing that 0 is an eigenvalue. 
We thus complete the first step of our project, which was presented (partly) in [4] by means of the formal asymptotic expansions coupled with numerical evidence, prior to the rigorous mathematical analysis due to [8] and the present paper. We conjecture that blow-up rate of a general blow-up solution of (1.6a) is the same as one of the particular solutions constructed in [8] and the present paper. See Remark 1.4 below for the detail.

Remark 1.4. As stated in [8, Remark 1.5], there is a striking analogy on singularity formation between equation (1.6a) and a semilinear heat equation $u_{t}=\Delta u+u^{p}, p>1$. For this equation Herrero and Velázquez [22,23] proved the existence of type II blow-up solutions $\left\{u_{\ell, \mathrm{HV}}\right\}_{\ell}$ if $N \geq 11, p>1+4 /(N-4-2 \sqrt{N-1})$, which play essential role in the classification of general blow-up solutions due to [27,28]. Analogous classification result was obtained for a different parabolic problem in [18] based on [19,32]. Taking account of these works, the authors expect that the blow-up solutions as in Theorem 1.1 and [8, Theorem 1.1] exhibit all possible blow-up mechanisms of (1.6a) for $d>d^{*}(k)$ with $k=1$. The same should be true also for $k \geq 2$, as [8, Theorem 1.1] is extended to the case $k \geq 2$ [33], though type I blow-up could occur in that case. The information about the number of intersections as stated in (iv) of Theorem 1.1 should be essential to claim such a statement.

Statement (ii) in Theorem 1.1 shows that the leading term near the singularity evolves in a non-self-similar scale due to the presence of function $\varepsilon(s)$, whereas statement (iii) implies that the solution behaves in a self-similar manner in the region $r \approx \sqrt{T-t}$. This fact is a key qualitative description of type II singularity. Based on the local estimates in Theorem 1.1, we may show that it applies also in its derivatives. Moreover, we may obtain the asymptotics of the energy density of the corresponding $k$-equivariant map.

Theorem 1.5. Assume the same hypotheses as in Theorem 1.1. Let $u$ and $\varepsilon(s)$ with $s=-\log (T-t)$ be as in Theorem 1.1 and let $\phi_{n_{0}}(y)$ be the function as in (1.16). Then

(i) Function $(\xi, t) \mapsto u(\xi \varepsilon(s) \sqrt{T-t}, t)$ is close to $U_{1}(\xi)$ in $C_{\text {loc }}^{2}[0, \infty)$ in the sense that

$$
\sum_{j=0}^{2} \sup _{\xi \leq K}\left|\frac{\partial^{j}}{\partial \xi_{j}}\left(u(\xi \varepsilon(s) \sqrt{T-t}, t)-U_{1}(\xi)\right)\right| \rightarrow 0 \quad \text { as } t \nearrow T
$$

for every $K>0$, and function $(y, t) \mapsto u(y \sqrt{T-t}, t)$ is close to $u^{*}(y, t):=\pi / 2-$ $D \varepsilon(s)^{\gamma} \phi_{n_{0}}(y)$ in $C_{\text {loc }}^{2}(0, \infty)$ in the sense that

$$
\sum_{j=0}^{2} \sup _{L \leq y \leq M}\left|\frac{\partial^{j}}{\partial y_{j}}\left(u(y \sqrt{T-t}, t)-u^{*}(y, t)\right)\right| \rightarrow 0 \quad \text { as } t \nearrow T
$$

for every $M>L>0$.

(ii) Let $F(x, t), F_{1}(x, t), F^{*}(x, t)$ be the $k$-equivariant maps defined by (1.4) corresponding to $u(r, t), U_{1}(r / \varepsilon(s) \sqrt{T-t}), u^{*}(r / \sqrt{T-t}, t)$ with $r=|x|$, respectively. Then:

$$
\sup _{\xi \leq K} \varepsilon(s)^{2}(T-t)\left|\left(|\nabla F|^{2}-\left|\nabla F_{1}\right|^{2}\right)(\xi \varepsilon(s) \sqrt{T-t}, t)\right| \rightarrow 0 \quad \text { as } t \nearrow T
$$


for every $K>0$, and

$$
\sup _{L \leq y \leq M} \frac{(T-t)}{\varepsilon(s)^{2 \gamma}}\left|\left(|\nabla F|^{2}-\left|\nabla F^{*}\right|^{2}\right)(y \sqrt{T-t}, t)\right| \rightarrow 0 \quad \text { as } t \nearrow T
$$

for every $M>L>0$.

Remark 1.6. Estimates (1.18) show that the leading term of the energy density $|\nabla F|^{2}$ in the designated regions are precisely given by $\left|\nabla F_{1}\right|^{2}$ and $\left|\nabla F^{*}\right|^{2}$, respectively, since we have

$$
\begin{aligned}
& A_{1} \leq \varepsilon(s)^{2}(T-t) \sup _{\xi \leq K}\left|\nabla F_{1}\right|^{2}(\xi \varepsilon(s) \sqrt{T-t}, t) \leq A_{2}, \\
& B_{1} \leq(T-t) \sup _{L \leq y \leq M}\left|\nabla F^{*}\right|^{2}(y \sqrt{T-t}, t) \leq B_{2}
\end{aligned}
$$

for some constants $A_{1}, A_{2}, B_{1}, B_{2}>0$. In particular, the energy density $|\nabla F|^{2}$ behaves in non-self-similar manner in the inner region $r \ll \varepsilon(s) \sqrt{T-t}$, but in self-similar manner in the self-similar region $r \approx \sqrt{T-t}$.

We just recall a general decay estimate essentially due to [15]. Suppose that initial data $u_{0} \equiv h_{0}$ satisfies $\left|h_{0}(r)\right| \leq M_{0}, r\left|h_{0}^{\prime}(r)\right| \leq M_{1}$ for a.e. $r>0$ for some constants $M_{0}, M_{1}>0$. Then any bounded solution $h$ of $(1.6 \mathrm{a})$ on $[0, T]$ with $h(\cdot, 0)=h_{0}$ satisfies

$$
\left|h_{r}(r, t)\right| \leq \frac{M_{2}}{r}, \quad r>0,
$$

uniformly on $[0, T]$, where $M_{2}>0$ is a constant depending only on $k, d, M_{0}$, and $M_{1}$. The proof of (1.20) is given in [15, Proposition B.1] for $k=1$ by using a heat kernel estimate, which works for any $k \geq 1$ without any change. Applying (1.20) as well as the identity (1.5) to our solution $u$ in the region $\{r>\varepsilon \sqrt{T-t}\}$, we immediately see that there holds $|\nabla F|^{2} \leq C / \varepsilon^{2}(T-t)$, whence the following corollary. In particular, the singularities of our solutions are categorized into the second kind in terms of Struwe's classification [36].

Corollary 1.7. Assume the same hypotheses as in Theorem 1.1. Then the energy density of $F$ enjoys the following blow-up rate estimate:

$$
\frac{C_{1}}{\varepsilon(s)^{2}(T-t)} \leq\|\nabla F(\cdot, t)\|_{L^{\infty}\left(\mathbb{R}^{d}\right)}^{2} \leq \frac{C_{2}}{\varepsilon(s)^{2}(T-t)},
$$

where $C_{1}, C_{2}>0$ are some constants, where $\varepsilon(s)$ is the function as in Theorem 1.1.

We finally mention a technical aspect of our approach. Our fundamental tool bases on a method of matched asymptotic expansions, which has been used in a number of nonlinear problems at least on a formal level, e.g, [4,13,34,37]. This method describes possible singularity mechanisms, especially locally in space, prior to verifying the actual existence of sought-for solutions. On the other hand, the justification of such formal asymptotic expansions is known to be a delicate problem, in general. In semilinear problems, a method of linearization about a stationary solution is commonly used. Information in 
the region away from the singularity is given by such a linearized problem. Such an approach has been adopted in various nonlinear parabolic problems, e.g, [19-21,31,35] after the pioneering work [22,23]. A related technique was used in [2]. In these works, the leading terms are obtained completely on the linear level. In subtler problems, such as the situation where 0 is an eigenvalue, deeper analysis is required. In particular, the situation becomes more complicated in a problem where a non-self-similar singularity can arise, because the standard method of higher-order approximation may not work. Some (but a few) works deal with such a situation [24,25] in the asymptotic methods, but the authors doubt if it would apply for our problem at least not directly. Instead, we adopt here the approach that has been recently developed in the second author's work [32]. See $\S \S \S 3.1 .1$ for detail.

The plan of the present article is as follows. In $\S 2$ we recall some preliminary results and revisit the formal construction of our sought-for solutions presented in [4]. A full proof of Theorem 1.1 is given in $\S 3$, which consists of six subsections. After some preparations in $\S \S 3.1$, the topological fixed-point argument is carried out in $\S \S 3.2$ by admitting a key a priori estimate. Consequently, Theorem 1.1 is proved. The remaining subsections of $\S 3$ are devoted to deriving a prior estimates. The most important one is about a bound of oscillation near the origin, presented in Lemma 3.6 in $\S \S 3.3$. This leads to sharp estimates suggested by the formal construction as demonstrated in $\S \S 3.4$ and $\S \S 3.5$. The section ends with the proof of Theorem 1.5 in $§ \S 3.6$.

\section{The formal construction}

In this section we formally derive the main results by means of the matched asymptotic expansions. Such a singularity mechanism was essentially found in [4]. We shall revisit and slightly modify the formal argument in that article, so as to estimate the magnitude of the remainder terms. No essential change on the most important terms from [4] appears in the final result but the reconstruction of the formal solution is convenient to prove the actual existence of the sought-for solution.

\subsection{Preliminary results}

To study the blow-up asymptotics around $(r, t)=(0, T)$, we use the self-similar variables:

$$
\begin{aligned}
& \Phi(y, s)=u(r, t), \\
& y=\frac{r}{\sqrt{T-t}}, \quad s=-\log (T-t) .
\end{aligned}
$$

In the following, let us write $\Lambda_{k}:=k(d+k-2)$ for simplicity. The new unknown function $\Phi$ then satisfies the rescaled equation:

$$
\Phi_{s}=\Phi_{y y}+\left(\frac{d-1}{y}-\frac{y}{2}\right) \Phi_{y}-\frac{\Lambda_{k}}{2 y^{2}} \sin (2 \Phi) \quad y>0,-\log T<s<+\infty,
$$

as well as the boundary condition $\Phi(0, s)=0$. We will construct a solution $f$ of equation (2.1c) that converges to $\pi / 2$ as $s \rightarrow \infty$ in an appropriate manner, setting

$$
v(y, s)=\Phi(y, s)-\frac{\pi}{2}
$$


Let us set

$$
\rho(y)=y^{d-1} \exp \left(-y^{2} / 4\right)
$$

It is readily seen that $v$ solves the equation

$$
\begin{aligned}
v_{s} & =v_{y y}+\left(\frac{d-1}{y}-\frac{y}{2}\right) v_{y}-\frac{\Lambda_{k}}{y^{2}} v+f(v) \\
& =\frac{1}{\rho} \frac{\partial}{\partial y}\left(\rho \frac{\partial v}{\partial y}\right)-\frac{\Lambda_{k}}{y^{2}} v+f(v) \equiv-\mathcal{A} v+f(v), \\
f(\psi) & =\frac{\Lambda_{k}}{2 y^{2}}(\sin (2 \psi)-2 \psi) .
\end{aligned}
$$

Let us write

$$
\begin{aligned}
& L_{r, \rho}^{2}\left(\mathbb{R}^{d}\right)=\left\{v \in L_{\mathrm{loc}}^{2}[0, \infty) ;\|v\|^{2}:=\int_{0}^{\infty} v^{2} \rho d y<\infty\right\}, \\
& H_{r, \rho}^{m}\left(\mathbb{R}^{d}\right)=\left\{v \in H_{\mathrm{loc}}^{m}[0, \infty) ;\|v\|_{H_{r, \rho}^{m}\left(\mathbb{R}^{d}\right)}^{2}:=\sum_{j=0}^{m} \sup _{|\alpha|=j}\left\|D_{y}^{\alpha} u\right\|^{2}<\infty\right\},
\end{aligned}
$$

where $m=1,2, \ldots$ These function spaces have Hilbert space structures endowed with canonical scalar products, respectively. When $d>d^{*}(k)$, a linearized operator $A$ : $L_{r, \rho}^{2}\left(\mathbb{R}^{d}\right) \rightarrow L_{r, \rho}^{2}\left(\mathbb{R}^{d}\right)$, that is initially defined in the set of smooth functions, may be extended to a unique self-adjoint operator (Friedrichs' extension) satisfying

$$
\langle A v, v\rangle \geq-\frac{\gamma}{2}\|v\|^{2}, \quad v \in D(A)
$$

where $\gamma$ is the positive constant defined in (1.9). The following lemma was obtained in [8, Lemma 3.4] for $k=1$ (cf. also [4]). The proof for $k \geq 2$ is entirely similar and thus omitted.

Proposition 2.1. Assume that $d>d^{*}(k)$ holds. Then the spectrum of $A$ consists only of simple eigenvalues $\left\{\lambda_{n}\right\}_{n=0}^{\infty} \subset \mathbb{R}$ with

$$
\lambda_{n}=n-\frac{\gamma}{2}, \quad n=0,1,2, \ldots,
$$

Eigenfunctions of $A$ associated to eigenvalues $\lambda_{n}$ are given by

$$
\phi_{n}(y)=\mathcal{N}_{n} y^{-\gamma} L_{n}^{(\omega / 2)}\left(\frac{y^{2}}{4}\right), \quad n=0,1,2, \ldots
$$

where $\omega=\omega_{k, d}, L_{n}^{(\nu)}$ denotes the standard associated Laguerre polynomial of order $\nu$, and

$$
\mathcal{N}_{n}=2^{-1-\omega / 2} \sqrt{\frac{n !}{\Gamma(n+1+\omega / 2)}}
$$

is a normalizing constant so that $\left\langle\phi_{n}, \phi_{m}\right\rangle=\delta_{n, m}$. Moreover,

$$
\begin{aligned}
& \phi_{n}(y)=c_{n} y^{-\gamma}(1+o(1)) \quad \text { as } y \rightarrow 0 ; \\
& \phi_{n}(y)=\tilde{c}_{n} y^{-\gamma+2 n}(1+o(1)) \quad \text { as } y \rightarrow \infty,
\end{aligned}
$$

where $c_{n}>0$ and $\tilde{c}_{n}$ are constants such that $(-1)^{n} \tilde{c}_{n}>0$ for $n=0,1,2, \ldots$ 
Remark 2.2 (The existence of neutral eigenvalues). It is convenient to make a list of triplets $(k, d, \gamma)$ for which an eigenvalue vanishes. Suppose that $\lambda_{n}=0$, i.e., $\gamma=2 n$, which requires, in view of $(1.9), \sqrt{(d-2)^{2}-4 k(d-2)-4 k^{2}}=d-2-4 n$. Under the assumption $d>d^{*}(k)$, the last identity is equivalent to

$$
\begin{aligned}
& (k-2 n)(d-2)+k^{2}+4 n^{2}=0, \\
& d-2-4 n>0 .
\end{aligned}
$$

As a matter of fact, there is an infinite number of triplet $(k, d, \gamma)$ that consists of integers satisfying (2.10). Up to $n=5$, the triplets $(k, d, \gamma)$ producing $\lambda_{n_{0}}=0$ are as follows:

$$
\begin{aligned}
& \lambda_{1}=0 \Longleftrightarrow(k, d, \gamma)=(1,7,2), \\
& \lambda_{2}=0 \Longleftrightarrow(k, d, \gamma)=(2,12,4),(3,27,4), \\
& \lambda_{3}=0 \Longleftrightarrow(k, d, \gamma)=(3,17,6),(4,28,6),(5,63,6), \\
& \lambda_{4}=0 \Longleftrightarrow(k, d, \gamma)=(4,22,8),(6,52,8),(7,115,8), \\
& \lambda_{5}=0 \Longleftrightarrow(k, d, \gamma)=(5,27,10),(6,36,10),(8,84,10),(9,183,10) .
\end{aligned}
$$

As already pointed out in $\S 1$, the both cases $2 \gamma>\omega$ and $2 \gamma<\omega$ can occur if $n_{0} \geq 2$.

Assumption 2.1. There exists a neutral eigenvalue: $\lambda_{n_{0}}=0$ for some $n_{0} \in \mathbb{N}$.

\subsection{Derivation of the formal asymptotics}

The asymptotic behavior (2.9a) of $\phi_{n}$ shows that the linearization in pointwise sense does not yield adequate first-order approximation for $y$ close to the origin. This fact indicates the onset of inner layer around the origin. We shall denote henceforth the size of inner layer by $\varepsilon(s)$ and assume that $\varepsilon(s)>0$ for all $s$ and

$$
\lim _{s \rightarrow \infty} \varepsilon(s)=\lim _{s \rightarrow \infty} \dot{\varepsilon}(s)=0 .
$$

To analyze the behavior in the inner layer we introduce new variables:

$$
\xi=\frac{y}{\varepsilon(s)}, \quad U(\xi, s)=\Phi(y, s)
$$

Equation (2.1c) then reads

$$
\varepsilon(s)^{2} U_{s}=U_{\xi \xi}+\left(\frac{d-1}{\xi}-\left(\varepsilon(s)^{2}-2 \varepsilon(s) \dot{\varepsilon}(s)\right) \frac{\xi}{2}\right) U_{\xi}-\frac{\Lambda_{k}}{2 \xi^{2}} \sin (2 U) .
$$

It is thus expected, to the leading order, $U(\xi, s)$ behaves asymptotically to a stationary solution of the original equation: $U(\xi, s) \sim U_{\alpha}(\xi)$ as $s \rightarrow \infty$ locally uniformly in $[0, \infty)$ for some $\alpha>0$. Here we just recall that the stationary solutions are given by a one-parameter family $\left\{U_{\alpha}\right\}$. Each function $U_{\alpha}$ is characterized by the boundary condition:

$$
U_{\alpha}(0)=0, \quad \lim _{\xi \rightarrow 0} \frac{U_{\alpha}(\xi)}{\xi^{k}}=\alpha^{k} .
$$

We shall just summarize some properties of stationary solutions. 
Proposition 2.3. Assume $d>d^{*}(k)$. Then any stationary solution $U_{\alpha}(\xi), \alpha>0$, of (1.6a) satisfying (2.11) is given by $U_{\alpha}(\xi)=U_{1}(\alpha \xi)$. Moreover, $U_{1}(\xi)$ has the following asymptotics:

$$
\begin{aligned}
U_{1}(\xi) & =\xi^{k}+O\left(\xi^{3 k}\right) \quad \text { as } \xi \rightarrow 0, \\
U_{1}^{\prime}(\xi) & =k \xi^{k-1}+O\left(\xi^{3 k-1}\right) \quad \text { as } \xi \rightarrow 0 ; \\
U_{1}(\xi) & =\frac{\pi}{2}-h \xi^{-\gamma}+O\left(\xi^{-\gamma-\delta}\right) \quad \text { as } \xi \rightarrow \infty, \\
U_{1}^{\prime}(\xi) & =h \gamma \xi^{-\gamma-1}+O\left(\xi^{-\gamma-\delta-1}\right) \quad \text { as } \xi \rightarrow \infty,
\end{aligned}
$$

where $\delta=\min \{2 \gamma, \omega\}$.

Proof. The first assertion is a consequence of uniqueness and scale-invariance. The proof of $(2.12 \mathrm{c})$ is found in $[4,26]$. One may easily prove $(2.12 \mathrm{~d})$ by using $(2.12 \mathrm{c})$ and the identity

$$
U_{1}^{\prime}(\xi)=\frac{k(d+k-2)}{2 \xi^{d-1}} \int_{0}^{\xi} r^{d-3} \sin \left(2 U_{1}(r)\right) d r, \quad \xi>0,
$$

with the help of H'Lôpital rule as well as the quadratic equation satisfied by $\gamma$. Similarly, we may derive (2.12a) and $(2.12 \mathrm{~b})$.

By normalization, we may suppose $U(\xi, s) \sim U_{1}(\xi)$ as long as $\varepsilon(s)^{2} \xi=o(1 / \xi)$, i.e., $y \ll 1$ as $s \rightarrow \infty$. We thus obtain

$$
\Phi(y, s) \sim \Phi_{\operatorname{inn}}(y, s):=U_{1}\left(\frac{y}{\varepsilon(s)}\right) \sim \frac{\pi}{2}-h \varepsilon(s)^{\gamma} y^{-\gamma}
$$

for $\varepsilon(s) \ll y \ll 1, s \rightarrow \infty$. Expansion (2.13) describes the approximation of out soughtfor solutions near the origin. We will describe another type of expansions valid for the outside the inner region. Let us expand the solution $v$ of (2.3) to a Fourier series:

$$
v(y, s)=a_{0}(s) \phi_{0}+a_{1}(s) \phi_{1}(y)+\cdots+a_{n_{0}}(s) \phi_{n_{0}}(r)+Q(y, s),
$$

where $\left\langle Q(\cdot, s), \phi_{0}\right\rangle=\cdots=\left\langle Q(\cdot, s), \phi_{n_{0}}\right\rangle=0$. Fourier coefficients $a_{n}, n=0,1, \ldots$, solve the ODE:

$$
\dot{a}_{n}(s)=-\lambda_{n} a_{n}(s)+\left\langle f(v(s)), \phi_{n}\right\rangle,
$$

where $f(v)$ is the function defined in (2.3).

As we have seen in Proposition 2.1, the eigenfunctions $\phi_{j}(y)$ behave as $y^{-\gamma}$ as $y \rightarrow 0$. To factor it out, we introduce the following dependent variable:

$$
W=y^{\gamma} v
$$

Equation (2.3a) then reads:

$$
\begin{aligned}
W_{s} & =W_{y y}+\left(\frac{m-1}{y}-\frac{y}{2}\right) W_{y}+\frac{\gamma}{2} W+g(W) \quad \text { with } \quad m=d-2 \gamma, \\
g(W) & =y^{\gamma} f\left(y^{-\gamma} W\right)=y^{\gamma-2} \frac{\Lambda_{k}}{2}\left\{\sin \left(2 y^{-\gamma} W\right)-2 y^{-\gamma} W\right\}
\end{aligned}
$$


(cf. (2.3)). Since $\gamma=2 n_{0}$ by Assumption 2.1, it turns out that $m$ is a positive integer. Hence the rescaled solution $W$ is canonically identified with a radial solution of $m$-dimensional equation. Let us define

$$
-\mathcal{L} V=V_{y y}+\left(\frac{m-1}{y}-\frac{y}{2}\right) V_{y}+\frac{\gamma}{2} V, \quad V \in \mathcal{D}(\mathcal{L}):=H_{r, \rho}^{2}\left(\mathbb{R}^{m}\right) .
$$

It is routine to see that the operator $\mathcal{L}$ is self-adjoint and its spectrum consists only of eigenvalues. Moreover, the $n$th eigenvalue is equal to $\lambda_{n}$, i.e., the $n$th eigenvalue of the operator $A$ defined before. Eigenfunctions $\psi_{n}$ of $\mathcal{L}$ associated to $\lambda_{n}$ are given by:

$$
\psi_{n}(y)=y^{\gamma} \phi_{n}(y) \text {. }
$$

Notice that $\lim _{y \rightarrow 0^{+}} \psi_{n}(y)=c_{n}$ exists for every $n$, where $c_{n}$ is the positive constant as in (2.9a). The following Fourier expansions are then canonically obtained from (2.14):

$$
W(y, s)=a_{0}(s) \psi_{0}+a_{1}(s) \psi_{1}(y)+\cdots+a_{n_{0}}(s) \psi_{n_{0}}(y)+y^{\gamma} Q(y, s) .
$$

Let $\phi$ be a continuous function on $\mathbb{R}_{+}$such that $\phi(y)=O\left(y^{-\gamma}\right)$ as $y \rightarrow 0$ and set $\psi(y):=y^{\gamma} \phi(y)$. A straightforward computation reveals that

$$
\langle g(W(\cdot, s)), \psi\rangle_{L_{r, \tilde{\rho}}^{2}\left(\mathbb{R}^{m}\right)}=\langle f(v(\cdot, s)), \phi\rangle_{L_{r, \rho}^{2}\left(\mathbb{R}^{d}\right)},
$$

where $\tilde{\rho}=\tilde{\rho}(y)=y^{m-1} e^{-y^{2} / 4}$. To estimate $a_{n}(s)$ we examine the above amount in detail. Let $L=L(s)$ be a continuous function satisfying $\varepsilon(s) \ll L \ll 1$ as $s \rightarrow \infty$. In order to see the contributions of inner and outer parts to $\langle g(W(\cdot, s))$, $\phi\rangle$, let us divide it as

$$
\begin{aligned}
\langle g(W(\cdot, s)), \psi\rangle & =\left(\int_{0}^{L}+\int_{L}^{\infty}\right) g(W(y, s)) \psi(y) y^{m-1} e^{-y^{2} / 4} d y \\
& =: I_{1}+I_{2} .
\end{aligned}
$$

It is crucial to determine the contributions of $I_{1}$ and $I_{2}$ as $s \rightarrow \infty$, which depend on $k$ and $d$ through the amounts $\omega$ and $\gamma$.

Since $v=\Phi(y, s)-\pi / 2 \sim \Phi_{\text {inn }}(y, s)-\pi / 2 \sim-h \varepsilon(s)^{\gamma} y^{-\gamma}$ as $y \rightarrow 0$, we obtain

$$
\begin{aligned}
I_{1} & =\frac{\Lambda_{k}}{2} \int_{0}^{L} \frac{1}{y^{2}}\{\sin (2 v)-2 v\} \psi(y) y^{m+\gamma-1} e^{-y^{2} / 4} d y \\
& \sim \frac{\Lambda_{k}}{2 \cdot 3 !} h^{3} \int_{0}^{L}\left(\frac{y}{\varepsilon(s)}\right)^{-3 \gamma} \psi(y) y^{d-3-\gamma} e^{-y^{2} / 4} d y \quad \text { as } L \rightarrow 0 .
\end{aligned}
$$

We then change the integral variable to the inner scale

$$
\xi=\frac{y}{\varepsilon(s)} \text {. }
$$

Since $d-3-4 \gamma=\omega-2 \gamma-1$, the last integral converges if and only if $\omega<2 \gamma$, whence:

$$
\begin{aligned}
I_{1} & \sim \begin{cases}\psi(0) \varepsilon(s)^{\gamma+\omega} \int_{0}^{\infty} G(\xi) \xi^{d-3-\gamma} d \xi & (\omega<2 \gamma), \\
\frac{\Lambda_{k}}{2 \cdot 3 !} \frac{h^{3}}{\omega-2 \gamma} \psi(0) \varepsilon(s)^{\gamma+\omega}\left(\frac{L}{\varepsilon(s)}\right)^{\omega-2 \gamma} & (\omega>2 \gamma),\end{cases} \\
G(\xi) & =\frac{\Lambda_{k}}{2}\left(\sin \left(2 U^{*}(\xi)-\pi\right)-\left(2 U^{*}(\xi)-\pi\right)\right)
\end{aligned}
$$

as $L \rightarrow 0$. 
Assumption 2.2. The leading term of $W(y, s)$ in the expansion $(2.18)$ is $a_{n_{0}}(s) \psi_{n_{0}}(y)$ and $\dot{\varepsilon}(s)=o(\varepsilon(s)) \quad$ as $s \rightarrow \infty$.

The inner expansion (2.13) requires

$$
a_{n_{0}}(s)=-\frac{h}{c_{n_{0}}} \varepsilon(s)^{\gamma}+o\left(\varepsilon(s)^{\gamma}\right)
$$

as $s \rightarrow \infty$. Taylor approximation yields

$$
I_{2} \sim-\frac{2 \Lambda_{k}}{3} \int_{L}^{\infty}\{W(y, s)\}^{3} \psi(y) y^{d-3-4 \gamma} e^{-y^{2} / 4} d y .
$$

Notice that the last integral diverges as $L \rightarrow 0$ if and only if $d-2<4 \gamma$. We thus obtain

$$
\begin{cases}I_{2}=O\left(\varepsilon(s)^{\gamma+\omega}\left(\frac{\varepsilon(s)}{L}\right)^{2 \gamma-\omega}\right)=o\left(\varepsilon(s)^{\gamma+\omega}\right) & (\omega<2 \gamma) \\ I_{2} \sim \frac{2 \Lambda_{k}}{3} h^{3} \varepsilon(s)^{3 \gamma} \int_{0}^{\infty}\left\{\psi_{n_{0}}(y)\right\}^{3} \psi(y) y^{d-3-4 \gamma} e^{-y^{2} / 4} d y & (\omega>2 \gamma)\end{cases}
$$

It then follows that

$$
\langle g(W(\cdot, s)), \psi\rangle \sim D \varepsilon(s)^{\gamma+\delta}
$$

with

$$
D= \begin{cases}\psi(0) \int_{0}^{\infty} G(\xi) \xi^{d-3-\gamma} d \xi & (\omega<2 \gamma) \\ -\frac{2 \Lambda_{k}}{3} h^{3} \int_{0}^{\infty}\left\{\psi_{n_{0}}(y)\right\}^{3} \psi(y) y^{d-3-4 \gamma} e^{-y^{2} / 4} d y & (\omega>2 \gamma)\end{cases}
$$

This last result suggests that the nonlinear term $g$ in (2.17) may be approximated by a Dirac mass supported at the origin of $\mathbb{R}^{m}$ when $\omega<2 \gamma$, whereas by a cubic function of $W$ when $\omega>2 \gamma$. We begin with the former case.

\subsubsection{The case $\omega<2 \gamma$}

Assumption 2.3. The nonlinear term $g(W)$ may be replaced by $\chi \varepsilon(s)^{\gamma+\omega} \delta(y)$ with

$$
\chi:=\frac{\Lambda_{k}}{2} \int_{0}^{\infty}\left[\sin \left(2 U^{*}(\xi)-\pi\right)-\left(2 U^{*}(\xi)-\pi\right)\right] \xi^{d-3-\gamma} d \xi
$$

Accordingly, the evolution of our sought-for solution is governed by equation

$$
W_{s}=-\mathcal{L} W+c(d) \chi \varepsilon(s)^{\gamma+\omega} \delta(y)
$$

in a weak sense, where $c(d)>0$ is a universal constant depending only on $d$.

Notice that since $G(\xi)$ is positive everywhere, so is $\chi$. Under the Assumption 2.3, the Fourier coefficients in (2.18) are approximated as

$$
a_{n}(s) \sim-\chi c_{n} \int_{s}^{\infty} e^{\lambda_{n}(\tau-s)} \varepsilon(\tau)^{\gamma+\omega} d \tau \quad \text { for } n=0,1, \ldots, n_{0},
$$


where $c_{n}>0$ is the constant as in (2.9a). As for the remainder term $y^{\gamma} Q(y, s)$, we expect

$$
y^{\gamma} Q(y, s) \sim c(d) \chi \varepsilon(s)^{\gamma+\omega} F(y)
$$

for some smooth function $F$ on $(0, \infty)$.

Under the Assumption 2.2, the function $F$ satisfies

$$
\begin{aligned}
-\left[F^{\prime \prime}+\left(\frac{m-1}{y}-\frac{y}{2}\right) F^{\prime}+\frac{\gamma}{2} F\right] & =\delta(y)-\sum_{k=0}^{n_{0}}\left\langle\delta, \psi_{k}\right\rangle \psi_{k}=: S(y), \\
\left\langle F, \psi_{j}\right\rangle & =0 \quad\left(j=0,1, \ldots, n_{0}\right) .
\end{aligned}
$$

After suitable approximation, we argue with classical Riesz-Schauder theory (cf. [32, $\S \S 3.1])$ to show that problem (2.27) has a unique solution, which is given by

$$
F(y)=\sum_{j \geq n_{0}+1} \frac{\left\langle S, \psi_{j}\right\rangle}{j-n_{0}} \psi_{j}(y)
$$

in the dual norm for a suitable weighted Sobolev space. Moreover, the asymptotic behavior of $F(y)$ as $y \rightarrow 0$ is determined by the Green function of the $m$-dimensional Laplace equation. Namely,

$$
F(y) \sim b_{0} y^{-(m-2)},
$$

where $b_{0}>0$ is a constant. Since $m-2=d-2 \gamma-2=\omega$, it follows that

$$
Q(y, s) \sim b_{0} c(d) \chi \varepsilon(s)^{\gamma+\omega} y^{-\gamma-\omega} \quad \text { as } y \rightarrow 0 .
$$

We have therefore obtained the following outer expansions:

$$
\Phi(y, s) \sim \frac{\pi}{2}-\chi \sum_{n=0}^{n_{0}} c_{n} \int_{s}^{\infty} e^{\lambda_{n}(\tau-s)} \varepsilon(\tau)^{\gamma+\omega} d \tau \cdot y^{-\gamma}+b_{0} c(d) \chi \varepsilon(s)^{\gamma+\omega} y^{-\gamma-\omega}
$$

as $y \rightarrow 0$. We just notice that the last term in the right-hand side of (2.29) is apparently large, but is in fact small in the intermediate region $\varepsilon(s) \ll y \ll 1$, as it can be seen at the points, for instance, $|y|=\varepsilon(s)^{\theta}$ with $\theta \in(0,1)$. Matching the both expansions $(2.13)$ and (2.29) in such a region, we obtain

$$
-h \varepsilon(s)^{\gamma}=-\chi \sum_{n=0}^{n_{0}-1} c_{n} \int_{s}^{\infty} e^{\lambda_{n}(\tau-s)} \varepsilon(\tau)^{\gamma+\omega} d \tau-\chi c_{n_{0}} \int_{s}^{\infty} \varepsilon(\tau)^{\gamma+\omega} d \tau .
$$

The asymptotic behavior of $\varepsilon(s)$ as $s \rightarrow \infty$ is then obtained by solving (2.30) asymptotically. A dominance balance argument then shows that the first term in the right is negligible. Hence:

$$
\varepsilon(s)^{\gamma} \sim \frac{\chi c_{n_{0}}}{h} \int_{s}^{\infty} \varepsilon(\tau)^{\gamma+\omega} d \tau
$$

as $s \rightarrow \infty$. An equivalent asymptotic differential equation is

$$
\dot{\varepsilon}(s) \sim-\frac{\chi c_{n_{0}}}{h \gamma} \varepsilon(s)^{1+\omega},
$$


which agrees with equation (45) in [4] with $\lambda_{N}=0$ there. We then solve this equation asymptotically, to get

$$
\varepsilon(s) \sim \varepsilon_{0}(s):=\frac{K_{1}}{s^{1 / \omega}} \quad \text { as } s \rightarrow \infty,
$$

where $K_{1}=h \gamma / \chi c_{n_{0}}>0$ is a constant. Scaling back to the original variables, we obtain

$$
\begin{aligned}
& u(r, t) \sim U_{1}\left(\frac{r}{\varepsilon(s) \sqrt{T-t}}\right) \quad \text { for } r \ll \varepsilon(s) \sqrt{T-t}, \\
& u(r, t) \sim \frac{\pi}{2}-\frac{h}{c_{n_{0}}} \varepsilon(s)^{\gamma} \phi_{n_{0}}\left(\frac{r}{\sqrt{T-t}}\right) \quad \text { for } \varepsilon(s) \sqrt{T-t} \ll r .
\end{aligned}
$$

\subsubsection{The case $\omega>2 \gamma$}

In this case the approximation of $g(W)$ by Dirac mass does not yield the first order approximation, but instead the cubic approximation dominates:

$$
g(W) \sim g_{\mathrm{cub}}(W):=-b y^{-2 \gamma-2} W^{3} \chi_{\{y \geq L\}} \text { with } b=\frac{2 \Lambda_{k}}{3},
$$

where $\chi_{A}$ stands for the indicator function of set $A$. The dependence of function $g_{\text {cub }}(W)$ on the space variable $y$ has not been specified explicitly for simplicity. Assumption 2.3 should be then replaced by:

Assumption 2.4. The nonlinear term $g(W)$ may be replaced by $g_{c u b}(W)$. Accordingly, the evolution of our sought-for solution $W$ is governed by equation

$$
W_{s}=-\mathcal{L} W+g_{\text {cub }}(W) .
$$

Assumption 2.2 is kept as is. Consider expansion (2.18). It follows from (2.25) and Assumption 2.4 that

$$
a_{n}(s) \sim D_{n} \int_{s}^{\infty} e^{\lambda_{n}(\tau-s)} \varepsilon(\tau)^{3 \gamma} d \tau \quad \text { for } n=0,1, \ldots, n_{0},
$$

where $D_{n}$ is the constant obtained by substituting $\psi=\psi_{n}$ in (2.25). More explicitly,

$$
\begin{aligned}
a_{n}(s) & =O\left(\varepsilon(s)^{3 \gamma}\right) \text { for } n=0, \ldots, n_{0}-1, \\
a_{n_{0}}(s) & =-C \varepsilon(s)^{\gamma}(1+o(1)) \text { with some constant } C>0 .
\end{aligned}
$$

Notice that the former estimate is due to integration by parts and Assumption 2.2, whereas the latter one is due to the matching condition required by the inner expansion (2.13). As for the remainder term $y^{\gamma} Q(y, s)$, we expect, up to higher order terms,

$$
y^{\gamma} Q(y, s) \sim \varepsilon(s)^{\ell} F(y)
$$

for some smooth function $F$ on $(0, \infty)$ and $\ell \geq \gamma$. The function $F$ should then satisfy

$$
\begin{aligned}
\ell \varepsilon(s)^{\ell-1} \dot{\varepsilon}(s) F & =-\varepsilon(s)^{\ell} \mathcal{L} F+g_{\text {cub }}(W)-\sum_{n=0}^{n_{0}}\left\langle g_{\text {cub }}(W), \psi_{n}\right\rangle \psi_{n}, \\
\left\langle F, \psi_{n}\right\rangle & =0 \text { for } n=0, \ldots, n_{0} .
\end{aligned}
$$


Taking the inner product with $\psi_{i}\left(i=n_{0}+1, \ldots\right)$ in $(2.37)$ and using $(2.25)$, we obtain

$$
\ell \varepsilon(s)^{\ell-1} \dot{\varepsilon}(s)\left\langle F, \psi_{i}\right\rangle=-\lambda_{i} \varepsilon(s)^{\ell}\left\langle F, \psi_{i}\right\rangle+D_{n} \varepsilon(s)^{3 \gamma}
$$

up to higher order terms. Suppose that $\ell<3 \gamma$ holds. The last equation then implies $\left\langle F, \psi_{i}\right\rangle=0$ for all $i=0,1, \ldots$, whence $F \equiv 0$. Suppose next that $\ell=3 \gamma$ holds. It then follows from (2.37) that, to the leading term,

$$
F^{\prime \prime}+\left(\frac{m-1}{y}-\frac{y}{2}\right) F^{\prime}+\frac{\gamma}{2} F=h^{3} b y^{-2 \gamma-2}(1+o(1)), \quad L<y \ll 1 .
$$

Hence there holds $F(y) \sim-B y^{-2 \gamma}$ as $y \rightarrow 0$ with $y>L$, where $B=h^{3} b / 2 \gamma(\omega-2 \gamma)>0$. Retuning to the self-similar variables, we obtain the following outer expansions:

$$
\Phi(y, s) \sim \frac{\pi}{2}-\left[\sum_{n=0}^{n_{0}} c_{n} D_{n} \int_{s}^{\infty} e^{\lambda_{n}(\tau-s)} \varepsilon(\tau)^{3 \gamma} d \tau+\frac{h^{3} b}{2 \gamma(\omega-2 \gamma)} \varepsilon(s)^{3 \gamma} y^{-2 \gamma}\right] y^{-\gamma}
$$

for $\varepsilon(s) \ll y \ll 1, s \rightarrow \infty$, where $c_{n}>0$ are the constants as in (2.9a). Matching the inner and outer expansions $(2.13)$ and $(2.39)$ in an intermediate region $\{\varepsilon(s) \ll y \ll 1\}$ and using (2.36), we obtain

$$
-h \varepsilon(s)^{\gamma}=-c_{n_{0}} D_{n_{0}} \int_{s}^{\infty} \varepsilon(\tau)^{3 \gamma} d \tau+o\left(\varepsilon(s)^{\gamma}\right)
$$

The asymptotic behavior of $\varepsilon(s)$ as $s \rightarrow \infty$ is then obtained by solving the ODE corresponding to (2.40) asymptotically, which essentially agrees with equation (45) in [4]. The result is:

$$
\varepsilon(s) \sim \varepsilon_{0}(s):=A s^{-1 / 2 \gamma} \text { with } A=\left(\frac{2 c_{n_{0}} D_{n_{0}}}{h}\right)^{-1 / 2 \gamma}
$$

as $s \rightarrow \infty$, whence the result.

\section{Full construction}

In this section we shall prove the actual existence of blow-up solutions that behave like the formal solutions constructed in $\S 2$. Let $\theta \in(0,1)$ be a constant satisfying

$$
\begin{array}{ll}
\theta<\frac{1}{2} \min \left\{\frac{2 \gamma-\omega}{2 \gamma-\omega+2}, \frac{\gamma}{\gamma+2}\right\} & (2 \gamma>\omega), \\
\theta<\frac{1}{2} \min \left\{\frac{\omega-2 \gamma}{\omega-2 \gamma+2}, \frac{\gamma}{\gamma+2}\right\} & (2 \gamma<\omega) .
\end{array}
$$

We now state our main results in the self-similar variables as follows:

Theorem 3.1. Assume that $d>d^{*}(k)$. Suppose that $\lambda_{n_{0}}$ vanishes, i.e., $\gamma=2 n_{0}$ for some $n_{0} \geq 1$. Let

$$
\varepsilon_{0}(s) \equiv\left\{\begin{array}{cc}
K_{1} s^{-1 / \omega} & (2 \gamma>\omega) \\
A s^{-1 / 2 \gamma} & (2 \gamma<\omega)
\end{array}\right.
$$


where $K_{1}$ and $A$ are the positive constants as in (2.33) and (2.41), respectively. Then there exist initial data $\Phi_{0} \in L^{\infty}\left(\mathbb{R}^{+}\right)$with $\Phi_{0}(0)=0$ and a positive smooth function $\varepsilon(s)$, that depends on the order of $2 \gamma$ and $\omega$, satisfying

$$
\left|\varepsilon(s)-\varepsilon_{0}(s)\right| \leq C \varepsilon_{0}(s)^{1+2 \theta}
$$

with some constant $C>0$ such that the solution $\Phi$ of $(2.1 \mathrm{c})$ with $\Phi\left(\cdot, s_{0}\right)=\Phi_{0}$ fulfills

$$
\begin{aligned}
& \left|\Phi(y, s)-U_{1}\left(\frac{y}{\varepsilon(s)}\right)\right|<\varepsilon(s)^{\theta} H\left(\frac{y}{\varepsilon(s)}\right) \quad \text { for } y \leq \varepsilon_{0}(s)^{\theta} \\
& \left|\Phi(y, s)-\frac{\pi}{2}+\frac{h}{c_{n_{0}}} \varepsilon(s)^{\gamma} \phi_{n_{0}}(y)\right|<\varepsilon(s)^{\gamma+2 \theta} y^{-\gamma}\left(1+y^{2 n_{0}+1}\right) \\
& \quad \text { for } \varepsilon_{0}(s)^{\theta} \leq y<\infty, s_{0} \leq s<\infty .
\end{aligned}
$$

Here $H(\xi)$ is a positive smooth function on $\mathbb{R}^{+}$satisfying

$$
H(\xi)= \begin{cases}O\left(\xi^{k}\right) & \text { as } \xi \rightarrow 0 \\ O\left(\xi^{-\gamma}\right) & \text { as } \xi \rightarrow \infty\end{cases}
$$

In particular, there holds

$$
\lim _{s \rightarrow \infty} \frac{\varepsilon(s)^{k} \Phi(y, s)}{y^{k}}=1 \quad \text { uniformly on the set }\left\{y \leq \varepsilon_{0}(s)^{\theta}\right\} .
$$

Moreover, the graphs of $\Phi(y, s)$ and $\pi / 2$ intersect transversely exactly $n_{0}$ times:

$$
Z_{(0, \infty)}\left[\Phi(\cdot, s)-\frac{\pi}{2}\right]=n_{0}
$$

and all the zeros lie in $\left(R^{-1}, R\right)$ with some large fixed number $R>0$ for all $s \geq s_{0}$, where $Z_{(0, \infty)}$ stands for the number of zeros in $(0, \infty)$.

\subsection{Setting of initial data and a basic functional framework}

Let $\varepsilon_{0}(s)$ be the positive function as in $(3.2)$ and set $\eta(s)=\left\{\varepsilon_{0}(s)\right\}^{-(2 / 3)(\gamma+2 \theta)}$. Let $S(y)$ be a nonnegative smooth function such that $S(y)=\pi / 2$ at $y=2 \eta\left(s_{0}\right), \pi / 2<S(y)<\pi$ for $2 \eta\left(s_{0}\right)<y<4 \eta\left(s_{0}\right)^{3}$, and $S(y) \equiv \pi$ for $4 \eta\left(s_{0}\right)^{3} \leq y<\infty$. For a given parameter $\alpha=\left(\alpha_{0}, \ldots, \alpha_{n_{0}}\right) \in \mathbb{R}^{n_{0}+1}$, we define

$$
\tilde{\phi}_{n_{0}}(y)= \begin{cases}\frac{1}{\alpha_{n_{0}}}\left[U_{1}\left(\frac{y}{\varepsilon_{0}\left(s_{0}\right)}\right)-\frac{\pi}{2}-\sum_{j=0}^{n_{0}-1} \alpha_{j} \phi_{j}(y)\right] & \left(y \leq \varepsilon_{0}\left(s_{0}\right)^{2 \theta}\right) \\ \phi_{n_{0}}(y) & \left(\varepsilon_{0}\left(s_{0}\right)^{2 \theta}<y<2 \eta\left(s_{0}\right)\right) \\ -\frac{1}{\alpha_{n_{0}}}\left\{\sum_{j=0}^{n_{0}-1} \alpha_{j} \phi_{j}(y)-S(y)+\frac{\pi}{2}\right\} & \left(2 \eta\left(s_{0}\right)<y<\infty\right)\end{cases}
$$

and

$$
\Phi_{0}(y):=\frac{\pi}{2}+\sum_{j=0}^{n_{0}-1} \alpha_{j} \phi_{j}(y)+\alpha_{n_{0}} \tilde{\phi}_{n_{0}}(y)
$$


so that

$$
\Phi_{0}(y)= \begin{cases}U_{1}\left(\frac{y}{\varepsilon_{0}\left(s_{0}\right)}\right) & \left(y \leq \varepsilon_{0}\left(s_{0}\right)^{2 \theta}\right) \\ \frac{\pi}{2}+\sum_{j=0}^{n_{0}} \alpha_{j} \phi_{j}(y) & \left(\varepsilon_{0}\left(s_{0}\right)^{2 \theta}<y<2 \eta\left(s_{0}\right)\right) \\ S(y) & \left(2 \eta\left(s_{0}\right)<y<\infty\right) .\end{cases}
$$

Remark 3.2. Due to the choice of $S(y)$, the initial data has finite energy. In terms of the original variables, the initial data $u_{0}(|x|)$ satisfies

$$
u_{0}(0)=0, \quad \lim _{|x| \rightarrow \infty} u_{0}(|x|)=\pi
$$

and $\partial_{x} u_{0}(x) \equiv 0$ for all sufficiently large $|x|$. The actually required condition on $\Phi_{0}$ (thus on $u_{0}$ ) for $|y| \gg \eta\left(s_{0}\right)^{3}$ is merely boundedness, so $S(y) \equiv \pi$ there may be replaced by $S(y) \equiv n \pi$ with $n \in \mathbb{N} \cup\{0\}$. It means that $\Phi_{0}(y)$ gets free of the equator $\pi / 2$ near $\infty$.

In terms of $W$ variable, the initial data is rephrased as

$$
\begin{aligned}
W\left(y, s_{0}\right)=W_{0}(y)=y^{\gamma}\left[\Phi_{0}(y)-\frac{\pi}{2}\right] & =\sum_{j=0}^{n_{0}-1} \alpha_{j} \psi_{j}(y)+\alpha_{n_{0}} \tilde{\psi}_{n_{0}}(y) \\
& =\sum_{j=0}^{n_{0}-1} a_{j}\left(s_{0}\right) \psi_{j}(y)+y^{\gamma} E_{0}(y),
\end{aligned}
$$

where $a_{j}(s)=\langle W(\cdot, s), \psi\rangle$ and $\tilde{\psi}_{n_{0}}(y)=y^{\gamma} \tilde{\phi}_{n_{0}}(y)$. Then there holds

$$
\begin{aligned}
a_{j}\left(s_{0}\right) & =\alpha_{j}+\alpha_{n_{0}}\left\langle\tilde{\psi}_{n_{0}}, \psi_{j}\right\rangle, \quad j=0,1, \ldots, n_{0}-1, \\
a_{n_{0}}\left(s_{0}\right) & =\alpha_{n_{0}}\left\langle\tilde{\psi}_{n_{0}}, \psi_{n_{0}}\right\rangle .
\end{aligned}
$$

It is not difficult to check that

$$
\begin{aligned}
& \left|\alpha_{j}\right|\left|\left\langle\tilde{\phi}_{n_{0}}, \phi_{j}\right\rangle\right| \leq C \varepsilon\left(s_{0}\right)^{\gamma+2 m \theta}, \quad j=0,1, \ldots, n_{0}-1, \\
& \left|\alpha_{n_{0}}\right|\left|1-\left\langle\tilde{\phi}_{n_{0}}, \phi_{n_{0}}\right\rangle\right| \leq C \varepsilon\left(s_{0}\right)^{\gamma+2 m \theta} .
\end{aligned}
$$

Then we have

$$
\begin{aligned}
E_{0}(y) & =W_{0}(y)-\sum_{j=0}^{n_{0}} a_{j}\left(s_{0}\right) \psi_{j}(y) \\
& =-\alpha_{n_{0}} \sum_{j=0}^{n_{0}-1}\left\langle\tilde{\psi}_{n_{0}}, \psi_{j}\right\rangle \psi_{j}(y)+\alpha_{n_{0}}\left[\tilde{\psi}_{n_{0}}(y)-\psi_{n_{0}}(y)+\left\{1-\left\langle\tilde{\psi}_{n_{0}}, \psi_{n_{0}}\right\rangle\right\} \psi_{n_{0}}(y)\right],
\end{aligned}
$$

and

$$
\left|E_{0}(y)\right| \leq \begin{cases}C \varepsilon_{0}\left(s_{0}\right)^{\gamma} & \text { for }|y| \leq \varepsilon_{0}\left(s_{0}\right)^{\theta} \\ C \varepsilon_{0}\left(s_{0}\right)^{\gamma+4 \theta}\left(1+|y|^{2 n_{0}+1 / 2}\right) & \text { for } \varepsilon_{0}\left(s_{0}\right)^{\theta}<|y|<\infty\end{cases}
$$




\subsubsection{The case $2 \gamma>\omega$}

Let $\Phi(y, s)=\Phi(y, s ; \alpha)$ be the solution of $(2.1 \mathrm{c})$ with initial data $\Phi\left(\cdot, s_{0}\right)=\Phi_{0}$, defined for $s_{0} \leq s \leq s_{1}$ with some $s_{1}>s_{0}$. We then define a function $\varepsilon(s)=\varepsilon\left(s: s_{0}, s_{1}, \Phi\right)$ as a (unique) solution of

$$
\left\{\begin{array}{l}
\chi c_{n_{0}} \int_{s}^{\infty} \varepsilon(\tau)^{\gamma+\omega} d \tau+\varphi(s)=h \varepsilon(s)^{\gamma} \quad\left(s>s_{0}\right) \\
\varepsilon\left(s_{0}\right)=\varepsilon_{0}\left(s_{0}\right)
\end{array}\right.
$$

with

$$
\begin{gathered}
\varphi(s)= \begin{cases}\int_{s}^{s_{1}} Y(\varepsilon(\tau), \Phi(\cdot, \tau)) d \tau & \left(s_{0} \leq s \leq s_{1}\right) \\
0 & \left(s_{1}<s\right)\end{cases} \\
Y(\varepsilon, \Psi(\cdot, s)) \\
=\Lambda_{k} \int_{0}^{\varepsilon^{2 \theta}}\left\{\cos \left(2 U_{1}\left(\frac{y}{\varepsilon}\right)-\pi\right)-1\right\}\left\{\Psi(y, s)-U_{1}\left(\frac{y}{\varepsilon}\right)\right\} \psi_{n_{0}}(y) y^{d-\gamma-3} e^{-\frac{y^{2}}{4}} d y .
\end{gathered}
$$

The unique existence of a local solution is shown once we rewrite the integral equation (3.16) to an explicit differential equation. The function $\varphi(s)$ may be considered to be a small perturbation, so that the equation (3.16) should be a regular perturbation of the equation (2.31) in $\S 2$. Indeed, existence and uniqueness of a solution to (3.16) is guaranteed as long as $\left|s_{1}-s_{0}\right|$ small enough. Moreover, it can be extended to $\left[s_{0}, s_{1}\right]$ with any $s_{1}>s_{0}$ provided that $\Phi$ belongs to $\overline{\mathcal{A}\left(s_{0}, s_{1} ; 1\right)}$.

Notice that the as long as $\varphi(s)=o\left(\varepsilon(s)^{\gamma}\right)$ in (3.16), there holds

$$
\varepsilon(s) \sim \varepsilon_{0}(s) \quad \text { as } \quad s \rightarrow \infty
$$

and the asymptotic identity for the corresponding derivative holds as well (cf. Proposition 3.3). Our goal is to show that if $\Phi(y, s)$ is a solution of (2.1c), defined for $s_{0} \leq s \leq s_{1}$, that belongs to a certain functional framework, then $\Phi(y, s) \sim(y / \varepsilon(s))^{k}$ for $y \ll \varepsilon(s)$. This amounts to showing that the solution $\Phi$ may be approximated by the formal solution constructed in $\S 2.2$. This last task is accomplished by comparing equations

$$
W_{s}=W_{y y}+\left(\frac{m-1}{y}-\frac{y}{2}\right) W_{y}+\frac{\gamma}{2} W+g(W)
$$

with

$$
V_{s}=V_{y y}+\left(\frac{m-1}{y}-\frac{y}{2}\right) V_{y}+\frac{\gamma}{2} W+c(d) \chi \varepsilon(s)^{\gamma+\omega} \delta(y)
$$

for the same initial data at $s=s_{0}$, where $g(W)=y^{\gamma} F\left(y^{-\gamma} W\right)$ and $\delta(y)$ denotes the Dirac mass supported at the origin of $\mathbf{R}^{m}, m=d-2 \gamma \in \mathbf{N}$. Equation (3.20) suggests another way of defining true size of the boundary layer:

$$
\varepsilon_{1}(s)^{\gamma}:=\frac{1}{c(d) \chi} \int_{0}^{\varepsilon_{0}(s)^{\theta}} f(v(y, s)) \phi(y) y^{d-1} e^{-y^{2} / 4} d y .
$$

As a matter of fact, $\varepsilon(s) \neq \varepsilon_{1}(s)$ in general. This idea was adopted by $[24,25]$ in different problems. We do not adopt this approach because it requires a priori pointwise estimates 
of the derivative $v_{s}(y, s)$, which is a hard task in general. Our approach, which comes from the second author's recent work [32], does not need such estimates, but instead sharp error estimates on approximate solutions in the inner regions. This last task is accomplished by computations based on asymptotic series expansions and the definition of $\varepsilon(s)$.

Let $\mathcal{A}\left(s_{0}, s_{1} ; \nu\right)(0<\nu \leq 1)$ be the set of all functions $\Phi(y, s)$ on $\overline{\mathbb{R}}_{+} \times\left[s_{0}, s_{1}\right]$ satisfying

$$
\begin{aligned}
& \left|\Phi(y, s)-U_{1}\left(\frac{y}{\varepsilon(s)}\right)\right|<\nu \varepsilon(s)^{\theta} H_{0}\left(\frac{y}{\varepsilon(s)}\right) \quad \text { for } y \leq \varepsilon_{0}(s)^{\theta} ; \\
& \left|\Phi(y, s)-\frac{\pi}{2}+h \varepsilon(s)^{\gamma} \phi_{n_{0}}(y)\right|<\nu \varepsilon(s)^{\gamma+2 \theta} y^{-\gamma}\left(1+y^{2 n_{0}+1}\right) \\
& \quad \text { for } \varepsilon_{0}(s)^{\theta} \leq y<\infty, s_{0} \leq s \leq s_{1} .
\end{aligned}
$$

We now define $\mathcal{U}_{s_{0}, s_{1}} \subset \mathbb{R}^{n_{0}+1}$ as the open subset of all points $\alpha=\left(\alpha_{0}, \ldots, \alpha_{n_{0}}\right) \in \mathbb{R}^{n_{0}+1}$ satisfying

$$
\left|\alpha_{j}\right|<\varepsilon_{0}\left(s_{0}\right)^{\gamma+2 \theta} \quad\left(j=0, \ldots, n_{0}-1\right), \quad\left|\alpha_{n_{0}}+\frac{h}{c_{n_{0}}} \varepsilon_{0}\left(s_{0}\right)^{\gamma}\right|<\varepsilon_{0}\left(s_{0}\right)^{\gamma+2 \theta}
$$

and

$$
\Phi(y, s ; \alpha) \in \mathcal{A}\left(s_{0}, s_{1}, 1\right)
$$

We note that

$$
\mathcal{U}_{s_{0}, s_{0}}=\left\{\alpha \in \mathbb{R}^{n_{0}+1}: \alpha=\left(\alpha_{0}, \ldots, \alpha_{n_{0}}\right) \text { satisfies }(3.24)\right\}
$$

and is nonempty. By a continuous dependence results, one has

$$
\mathcal{U}_{s_{0}, s_{1}} \neq \emptyset \quad \text { whenever } \quad\left(s_{1}-s_{0}\right) \quad \text { is sufficiently small. }
$$

\subsubsection{The case $2 \gamma<\omega$}

Let $\theta^{\prime} \in(0,1)$ be a constant such that $\theta^{\prime}>2 \theta$. We denote by $g_{L}(W)$ the leading term in the set $\left\{y>\varepsilon_{0}(s)^{\theta^{\prime}}\right\}$ of the nonlinear term $g(W)$ as a function of $W$ :

$$
g_{L}(W)=-b y^{-2 \gamma-2} W^{3} \chi_{\left\{y>\varepsilon_{0}(s)^{\theta^{\prime}}\right\}} .
$$

For a given function $\Phi$, we define a function $\varepsilon(s)$ as a solution of

$$
\begin{aligned}
& \frac{h}{c_{n_{0}}} \varepsilon(s)^{\gamma}=\chi\left(\frac{h}{c_{n_{0}}}\right)^{3} \int_{s}^{\infty} \varepsilon(\tau)^{3 \gamma} d \tau-\tilde{\varphi}(s) \quad\left(s>s_{0}\right), \\
& \tilde{\varphi}(s)= \begin{cases}\int_{s}^{s_{1}} \tilde{Y}(\varepsilon(\tau), \Phi(\cdot, \tau)) d \tau & \left(s_{0} \leq s \leq s_{1}\right) \\
0 & \left(s_{1}<s\right),\end{cases} \\
& \tilde{Y}(\varepsilon, \Phi(\cdot, s))=\left\langle g_{L}^{\prime}\left(W_{\text {out }}\right)\left(W-W_{\text {out }}\right) \chi_{\left\{y>\varepsilon_{0}(s)^{\theta^{\prime}}\right\}}, \psi_{n_{0}}\right\rangle \\
& W_{\text {out }}(y, s):=a_{n_{0}}^{*}(s) \psi_{n_{0}}(y):=-\frac{h}{c_{n_{0}}} \varepsilon(s)^{\gamma} \psi_{n_{0}}(y) \text {. }
\end{aligned}
$$

such that $\varepsilon\left(s_{0}\right)=\varepsilon_{0}\left(s_{0}\right)$, where ' denotes the differentiation with respect to $W$. The setting of initial data, the definitions of $\mathcal{A}\left(s_{0}, s_{1} ; \nu\right)$ and $\mathcal{U}_{s_{0}, s_{1}}$ are exactly the same as of $\S \S 3.1$ except for the definitions of $\varepsilon_{0}(s)$ and $\varepsilon(s)$. 


\subsection{Topological arguments}

We begin with showing that the positive function $\varepsilon(s)$ defined in (3.16) (or respectively (3.26)) behaves as $\varepsilon_{0}(s)$ (cf. (3.2)) to the leading term as well as its first derivative.

Proposition 3.3. Suppose that a solution $\Phi$ of (2.1c) belongs to $\overline{\mathcal{A}\left(s_{0}, s_{1} ; 1\right)}$. Then there exist constants $D, D^{\prime}>0$ such that

$$
\begin{aligned}
& \left|\varepsilon(s)-\varepsilon_{0}(s)\right| \leq D \varepsilon_{0}(s)^{1+\theta} \\
& \left|\dot{\varepsilon}(s)-\dot{\varepsilon}_{0}(s)\right| \leq D^{\prime} \varepsilon_{0}(s)^{1+\omega+\theta}
\end{aligned}
$$

for $s_{0} \leq s \leq s_{1}$. Moreover, $\dot{\varepsilon}(s)$ is Hölder continuous in $\left(s_{0}, s_{1}\right]$ for every $\alpha \in(0,1)$ and its Hölder norm is locally bounded in $\left(s_{0}, s_{1}\right]$ uniformly with respect to $s_{1}$.

Proof. We shall state the proof only for the case of $2 \gamma>\omega$ since the case of $2 \gamma<\omega$ is entirely similar. The membership of $\Phi$ to $\overline{\mathcal{A}\left(s_{0}, s_{1} ; 1\right)}$ and the elementary inequality $1-\cos x \leq 2^{-1} x^{2}(x \in \mathbb{R})$ imply

$$
\begin{aligned}
|Y(\varepsilon, \Phi(\cdot, s))| & \leq C \varepsilon(s)^{\theta+\gamma+\omega} \int_{0}^{\varepsilon(s)^{\theta-1}}\left\{2 U_{1}(\xi)-\pi\right\}^{2} H_{0}(\xi) \xi^{\gamma+\omega-1} d \xi \\
& \leq C \varepsilon(s)^{\theta+\gamma+\omega} .
\end{aligned}
$$

Boundedness of the last integral is a consequence of the asymptotics $(2.12 \mathrm{c})$ of $U_{1}(\xi)$ and $H_{0}(\xi) \sim h \gamma \xi^{-\gamma}$ as $\xi \rightarrow \infty$ and the assumption $2 \gamma>\omega$.

We now begin with the proof of the estimates in (3.27). Differentiating the both sides of (3.16), we obtain $-\chi c_{n_{0}} \varepsilon(s)^{\gamma+\omega}-Y(\varepsilon, \Phi(\cdot, s))=h \gamma \varepsilon(s)^{\gamma-1} \dot{\varepsilon}(s)$, whence:

$$
\begin{aligned}
\dot{\varepsilon}(s) & =-\frac{\chi c_{n_{0}}}{h \gamma} \varepsilon(s)^{1+\omega}-\frac{Y(\varepsilon, \Phi(\cdot, s))}{h \gamma \varepsilon(s)^{\gamma-1}} \\
& \equiv-a \varepsilon(s)^{1+\omega}\{1+f(\varepsilon(s))\},
\end{aligned}
$$

where $a=\chi c_{n_{0}} / h \gamma>0$. Since $|f(\varepsilon(s))| \leq C \varepsilon(s)^{\theta}$ and $\dot{\varepsilon}_{0}(s)=-a \varepsilon_{0}(s)^{1+\omega}$ with $\varepsilon\left(s_{0}\right)=$ $\varepsilon_{0}\left(s_{0}\right)$, it is rather easy to show that $\dot{\varepsilon}(s)<0$ and the following rough bounds hold:

$$
\frac{1}{2} \varepsilon_{0}(s)<\varepsilon(s)<\frac{3}{2} \varepsilon_{0}(s) \quad \text { for } s_{0} \leq s \leq s_{1}
$$

as long as $\varepsilon\left(s_{0}\right)$ is small enough. Taking a difference between the identities satisfied by $1 / \varepsilon(s)^{\omega}$ and $1 / \varepsilon_{0}(s)^{\omega}$ and using $\varepsilon\left(s_{0}\right)=\varepsilon_{0}\left(s_{0}\right)$, we obtain

$$
\frac{1}{\varepsilon(s)^{\omega}}-\frac{1}{\varepsilon_{0}(s)^{\omega}}=a \omega \int_{s_{0}}^{s} f(\varepsilon(\tau)) d \tau
$$

for $s_{0} \leq s \leq s_{1}$. We now exploit the elementary inequality $\left|t_{1}^{1 / \omega}-t_{2}^{1 / \omega}\right| \leq\left|t_{1}-t_{2}\right|$. $\min \left\{t_{1}, t_{2}\right\}^{1 / \omega-1}\left(t_{1}, t_{2}>0\right)$ and use (3.31), to get

$$
\left|\varepsilon(s)-\varepsilon_{0}(s)\right| \leq a \omega \varepsilon_{0}(s) \varepsilon(s)^{\omega} \int_{s_{0}}^{s}|f(\varepsilon(\tau))| d \tau .
$$


Since $\omega \geq 1>\theta$, the error estimate $|f(\varepsilon(s))| \leq C \varepsilon(s)^{\theta}$ and the rough bound (3.30) yield

$$
\int_{s_{0}}^{s}|f(\varepsilon(\tau))| d \tau \leq C^{\prime} \varepsilon_{0}(s)^{\theta-\omega}
$$

for some constant $C^{\prime}>0$. The first claim (3.27a) then follows from (3.32) and (3.33).

We then proceed to show the second claim (3.27b). Taking a difference in the differential equations satisfied by $\varepsilon(s)$ and $\varepsilon_{0}(s)$, we obtain, by (3.28),

$$
\begin{aligned}
\left|\dot{\varepsilon}(s)-\dot{\varepsilon}_{0}(s)\right| & \leq a\left|\varepsilon(s)^{1+\omega}-\varepsilon_{0}^{1+\omega}(s)\right|+|Y(\varepsilon, \Phi(\cdot, s))| \\
& \leq a \varepsilon(s)^{\omega}\left|\varepsilon(s)-\varepsilon_{0}(s)\right|+C \varepsilon(s)^{1+\omega+\theta} .
\end{aligned}
$$

The desired estimate then follows from the first claim (3.27a) and (3.30).

We next estimate a Hölder norm of $\dot{\varepsilon}(s)$. To this end, it suffices to show:

For every $\alpha \in(0,1), \nu>0$ there is a constant $C>0$ independent of $s_{1}$ such that

$$
\mid Y(\varepsilon(s), \Phi(\cdot, s))-Y\left(\varepsilon\left(s^{\prime}, \Phi\left(\cdot, s^{\prime}\right)\right)\left|\leq C \varepsilon(s)^{\gamma-1}\right| s-\left.s^{\prime}\right|^{\alpha}, \quad{ }^{\forall} s, s^{\prime} \in\left[s_{0}+\nu, s_{1}\right] .\right.
$$

We are going to show this claim by applying classical parabolic estimates for equation (2.1c). Suppose firstly $k \geq 2$. Then for any $p>d+1$ there is a constant $C_{p}>0$ such that

$$
\int_{s}^{s+1} d \tau \int_{0}^{1}\left|\frac{\sin (2 \Phi)}{y^{2}}\right|^{p} y^{d-1} d y \leq C_{p, k} \varepsilon(s)^{d-2 p} .
$$

This is readily seen by splitting the space integral as $\int_{0}^{1}=\int_{0}^{\varepsilon}+\int_{\varepsilon}^{1}$ with $\varepsilon=\varepsilon(s)$ and using the bound $|\Phi(y, s)| \leq 2(y / \varepsilon)^{k}$ for $y \leq 1$. Set $Q=B_{1} \times\left(s_{0}, s_{1}\right]$ with $B_{r}=\{|y|<r\}$. Then, due to $L^{p}$ theory, for any $\nu \in(0,1)$ there exists a constant $M>0$ such that

$$
\|\Phi\|_{W_{p}^{2,1}(Q(\nu))} \leq M\left(\|\Phi\|_{L^{p}(Q)}+C_{d, p, k} \varepsilon(s)^{d-2 p}\right),
$$

where $Q(\nu)=B_{1 / 2} \times\left(s_{0}+\nu, s_{1}\right]$. Notice that $\|\Phi\|_{L^{p}(Q)}$ is bounded by a positive constant depending only on $\left\|u_{0}\right\|_{L^{\infty}(0, \infty)}$. By a version of Sobolev inequality [1, Lemma 4.28, IV], we have

$$
\|\Phi\|_{C^{\alpha, \alpha / 2}(\overline{Q(\nu)})} \leq C(d, p, k, \nu, M) \varepsilon(s)^{d / p-2},
$$

where $C^{\lambda, \lambda / 2}(\bar{Q})$ stands for the standard Hölder spaces of order $\lambda \in(0,1)$ in $\bar{Q}$ with respect to parabolic distance. It then follows from this and (2.12c) that, for $s, s^{\prime} \in\left[s_{0}+\nu, s_{1}\right]$,

$$
\begin{aligned}
& \int_{0}^{\varepsilon(s)^{\theta}}\left|2 U_{1}\left(\frac{y}{\varepsilon(s)}\right)-\pi\right|^{2}\left|\Phi(y, s)-\Phi\left(y, s^{\prime}\right)\right| y^{\omega+\gamma-1} d y \\
\leq & M\|\Phi\|_{C^{\alpha, \alpha / 2}(\overline{Q(\nu)})}\left|s-s^{\prime}\right|^{\alpha / 2} \int_{0}^{\varepsilon(s)^{\theta}}\left|2 U_{1}\left(\frac{y}{\varepsilon(s)}\right)-\pi\right|^{2} y^{\omega+\gamma-1} d y \\
\leq & C_{\nu}\left|s-s^{\prime}\right|^{\alpha / 2} \varepsilon(s)^{d / p-2} \varepsilon(s)^{\omega+\gamma} \int_{0}^{\varepsilon(s)^{\theta-1}}\left|2 U_{1}(\xi)-\pi\right|^{2} \xi^{\omega+\gamma-1} d \xi \ll \varepsilon(s)^{\gamma-1}\left|s-s^{\prime}\right|^{\alpha / 2}
\end{aligned}
$$

since $\omega \geq 1$. The other terms arising when evaluating the difference $Y(s, \Phi(\cdot, s))-$ $Y\left(s^{\prime}, \Phi\left(\cdot, s^{\prime}\right)\right)$ are easily estimated, whence the result. In the case $k=1$, the tuple satisfying our basic assumption is $(d, \gamma, \omega)=(7,2,1)$. We change the dependent variable as $\Psi(y, s):=\Phi(y, s) / y^{k}$ and argue as before for the equation satisfied by $\Psi$. The detail is left to the reader (cf. the proof of Proposition 3.14 below for a related argument). 
Let

$$
\Psi_{j}(s) \equiv \begin{cases}\chi c_{j} \varepsilon(s)^{\gamma+\omega} & (2 \gamma>\omega) \\ \left\langle g_{L}\left(W_{\text {out }}(\cdot, s)\right), \psi_{j}\right\rangle & (2 \gamma<\omega) .\end{cases}
$$

We now define a continuous map $P\left(\cdot ; s_{1}\right): \mathbb{R}^{n_{0}+1} \rightarrow \mathbb{R}^{n_{0}+1}$, as

$$
\begin{aligned}
P\left(\alpha ; s_{1}\right) & =\left(p_{0}\left(\alpha ; s_{1}\right), \ldots, p_{n_{0}}\left(\alpha ; s_{1}\right)\right) \\
p_{j}\left(\alpha ; s_{1}\right) & =\left(\Phi\left(\cdot, s_{1} ; \alpha\right)-\frac{\pi}{2}, \phi_{j}\right)_{L_{\rho}^{2}\left(\mathbb{R}^{d}\right)}+\int_{s}^{\infty} e^{\lambda_{j}(\tau-s)} \Psi_{j}(\tau) d \tau .
\end{aligned}
$$

When $s_{1}=s_{0}$, we have

$$
P\left(\alpha ; s_{0}\right)=\alpha+\left(0, \ldots, 0, h \varepsilon_{0}\left(s_{0}\right)^{\gamma}\right)+O\left(\varepsilon_{0}\left(s_{0}\right)^{\gamma+2 \theta}\right)
$$

for any $\alpha \in \mathcal{U}_{s_{0}, s_{0}}$ as $s_{0} \rightarrow \infty$. Thus, in particular, $P\left(\alpha ; s_{0}\right)-\left(0, \ldots, 0, h \varepsilon_{0}\left(s_{0}\right)^{\gamma}\right)$ is a small perturbation of the identity map. Let deg stand for the mapping degree of $P\left(\cdot ; s_{1}\right)$ with respect to $0 \in \mathbb{R}^{n_{0}+1}$. The standard homotopy invariance property implies

$$
\operatorname{deg}\left(P\left(\cdot ; s_{0}\right), 0, \mathcal{U}_{s_{0}, s_{0}}\right)=1 .
$$

We also have $\operatorname{deg}\left(P\left(\cdot ; s_{1}\right), 0, \mathcal{U}_{s_{0}, s_{1}}\right)=1$ as long as $\mathcal{U}_{s_{0}, s_{1}} \neq \emptyset$.

Lemma 3.4. The set $\mathcal{U}_{s_{0}, s_{1}}$ is not empty for any $s_{1} \in\left[s_{0}, \infty\right)$.

Lemma 3.5. Suppose that $\alpha \in \mathbb{R}^{n_{0}+1}$ belong to the closure of $\mathcal{U}_{s_{0}, s_{1}}$. Then if $P\left(\alpha ; s_{1}\right)=0$, it turns out that $\Phi(y, s ; \alpha) \in \mathcal{A}\left(s_{0}, s_{1}, 1 / 2\right)$.

In order to keep the flow of the main arguments, we shall postpone the proof of Lemma 3.5 to $\S \S 3.5$ and admit the claim here.

Proof of Lemma 3.4. Suppose that

$$
s^{*}:=\sup \left\{s_{1}>s_{0}: \mathcal{U}_{s_{0}, s_{1}} \neq \emptyset\right\}<\infty .
$$

Then there exists a sequence $\left\{s_{n}\right\}_{n=0}^{\infty} \subset\left[s_{0}, \infty\right)$ such that $\mathcal{U}_{s_{0}, s_{n}} \neq \emptyset$ for each $n$ and $s_{n} \nearrow s^{*}$ as $n \rightarrow \infty$. Due to the homotopy invariance, there holds

$$
\operatorname{deg}\left(P\left(\cdot ; s_{n}\right), 0, \mathcal{U}_{s_{0}, s_{n}}\right)=\operatorname{deg}\left(P\left(\cdot ; s_{0}\right), 0, \mathcal{U}_{s_{0}, s_{0}}\right)=1 .
$$

Therefore there exists a root $\alpha_{n}$ of $P\left(\cdot ; s_{n}\right)$ in $\mathcal{U}_{s_{0}, s_{n}}$ for each $n$. Lemma 3.5 then guarantees that $\Phi\left(y, s ; \alpha_{n}\right)$ is in $\mathcal{A}\left(s_{0}, s_{n}, 1 / 2\right)$. Since $\left\{\alpha_{n}\right\}_{n=0}^{\infty} \subset \mathbb{R}^{n_{0}+1}$ is a bounded sequence, there is a convergent subsequence, still denoted by $\left\{\alpha_{n}\right\}_{n=0}^{\infty}$. Set $\alpha_{*}=\lim _{n \rightarrow \infty} \alpha_{n}$. In view of Proposition 3.3, the sequence $\left\{\varepsilon_{n}(s)\right\} \equiv\left\{\varepsilon\left(s, s_{0}, s_{n}, \Phi\left(\cdot ; \alpha_{n}\right)\right)\right\}$ satisfies

$$
\left|\dot{\varepsilon}_{n}(s)\right|+\varepsilon_{n}(s) \leq K \varepsilon_{0}(s)
$$

with some constant $K>0$ independent of $n$, thus converging, up to subsequence, to a continuous function $\varepsilon_{*}(s)$ uniformly in $\left[s_{0}, \infty\right)$ as $n \rightarrow \infty$. Let $\left\{\varphi_{n}(s)\right\}$ denote a sequence of the functions defined in (3.17) (or respectively, (3.26b)) with $\left(s_{1}, \Phi\right)$ replaced by $\left(s_{n}, \Phi\left(\cdot ; \alpha_{n}\right)\right)$ there. By continuous dependence on initial data and the dominated convergence theorem, we have $\lim _{n \rightarrow \infty} \varphi_{n}(s)=\varphi_{*}(s)$, where $\varphi_{*}(s)=\int_{s}^{s^{*}} Y\left(\tau, \Phi\left(\cdot, \tau ; \alpha^{*}\right)\right) d \tau$ 
for $s \leq s^{*}$ and $\varphi_{*}(s)=0$ for $s>s^{*}$ (cf. proof of Proposition 3.3). Consider the case $2 \gamma>\omega$. The function $\varepsilon_{*}(s)$ solves

$$
\chi c_{n_{0}} \int_{s}^{\infty} \varepsilon_{*}(\tau)^{\gamma+\omega} d \tau+\varphi_{*}(s)=h \varepsilon_{*}(s)^{\gamma}, \quad \varepsilon\left(s_{0}\right)=\varepsilon_{0}\left(s_{0}\right) .
$$

It then turns out that $\alpha_{*}$ belongs to $\overline{\mathcal{U}_{s_{0}, s^{*}}}$ and $P\left(\alpha^{*} ; s^{*}\right)=0$, whence $\Phi\left(y, s ; \alpha^{*}\right)$ is in $\mathcal{A}\left(s_{0}, s^{*}, 1 / 2\right)$ due to Lemma 3.5. Moreover, Corollary 3.8 below, (3.12), and (3.13) imply

$$
\left|\alpha_{j}^{*}\right|<\varepsilon_{0}\left(s_{0}\right)^{\gamma+3 \theta}\left(j=0, \ldots, n_{0}-1\right), \quad\left|\alpha_{n_{0}}^{*}+\frac{h}{c_{n_{0}}} \varepsilon_{0}\left(s_{0}\right)^{\gamma}\right|<\varepsilon_{0}\left(s_{0}\right)^{\gamma+3 \theta} .
$$

Therefore $\alpha^{*}$ lies in $\mathcal{U}_{s_{0}, s^{*}}$. A continuous dependence result then shows that there exists $\delta>0$ such that $\mathcal{U}_{s_{0}, s^{*}+\delta} \neq \emptyset$, which contradicts (3.40). The case $2 \gamma<\omega$ is similar and thus omitted. The proof is complete.

Proof of Theorems 3.1 and 1.1. Take a sequence $\left\{s_{n}\right\} \nearrow \infty$. Due to Lemma 3.4, there exists an $\alpha_{n} \in \mathcal{U}_{s_{0}, s_{n}}$ such that $P\left(\alpha_{n} ; s_{n}\right)=0$ for each $n$. Arguing as in the proof of Lemma 3.4 , we may prove that the tuple of $\varepsilon(s) \equiv \varepsilon\left(s ; s_{0}, \infty, \Phi\left(\cdot ; \alpha_{*}\right)\right)$ and $\Phi(y, s) \equiv \Phi\left(y, s ; \alpha_{*}\right)$ satisfies conditions (3.3)-(3.7). The claim (3.8) is a consequence of (3.5) and the wellknown zero-number diminishing property for one-dimensional parabolic equation applied to function $\Psi(y, s)=\Phi(y, s) / y^{k}$. Consequently, Theorem 3.1 is proved. Returning to the variable $(r, t)$ and $u$, we readily verify the claim of Theorem 1.1. The proof is complete.

\subsection{A priori estimates in the inner regions}

The goal of this subsection is to estimate possible oscillation of $\Phi(y, s)$ in the inner region, to be presented in Lemma 3.6 below. To this end, we prepare some notations. Let us set $V(\xi, s)=U(\xi, s)-U_{1}(\xi)$ with $U(\xi, s)=\Phi(\varepsilon(s) \xi, s)$. We have

$$
V(0, s)=0, \quad \lim _{\xi \rightarrow 0} \frac{V(\xi, s)}{\xi^{k}}=0 .
$$

The function $V$ solves

$$
\begin{aligned}
\varepsilon(s)^{2} V_{s}= & V_{\xi \xi}+\frac{d-1}{\xi} V_{\xi}-\mu(s) \frac{\xi}{2} V_{\xi}-\frac{\Lambda_{k}}{\xi^{2}} \cos \left(2 U_{1}\right) V- \\
& -\frac{\Lambda_{k}}{2 \xi^{2}}\left\{\sin \left(2 U_{1}+2 V\right)-\sin \left(2 U_{1}\right)-2 \cos \left(2 U_{1}\right) V\right\}-\mu(s) \frac{\xi}{2}\left(U_{1}\right)_{\xi},
\end{aligned}
$$

where, by (3.16),

$$
\begin{aligned}
\mu(s) & =\varepsilon(s)^{2}-2 \varepsilon(s) \dot{\varepsilon}(s) \\
& =\varepsilon(s)^{2}\left(1+O\left(\frac{1}{s}\right)\right) \quad \text { as } s \rightarrow \infty .
\end{aligned}
$$

Let us consider a related equation:

$$
\begin{aligned}
0 & =V_{0, \xi \xi}+\frac{d-1}{\xi} V_{0, \xi}-\frac{\Lambda_{k}}{\xi^{2}} \cos \left(2 U^{*}\right) V_{0}-\mu(s) T(\xi), \\
T(\xi) & :=\frac{\xi}{2}\left(U_{1}\right)_{\xi} .
\end{aligned}
$$


A solution $V(\xi, s)$ is given by $V(\xi, s)=\mu(s) H(\xi)$, where function $H(\xi)$ solves

$$
\begin{aligned}
H^{\prime \prime}+\frac{d-1}{\xi} H^{\prime}-\frac{\Lambda_{k}}{\xi^{2}} \cos \left(2 U_{1}\right) H & =T(\xi), \\
H(0) & =0, \quad H(\xi)=O\left(\xi^{k+2}\right) \quad \text { as } \xi \rightarrow 0 .
\end{aligned}
$$

Let $\bar{\alpha}>0$ be a constant. It is readily seen that function

$$
H_{0}(\xi):=\left.\partial_{\alpha} U_{\alpha}(\xi)\right|_{\alpha=\bar{\alpha}}=\xi U_{1}^{\prime}(\bar{\alpha} \xi)
$$

is a solution of the homogeneous equation with $\cos \left(2 U_{1}\right)$ replaced by $\cos \left(2 U_{\bar{\alpha}}\right)$ satisfying

$$
H_{0}(0)=0, \quad H_{0}(\xi)=(\bar{\alpha} \xi)^{k}(1+o(1)) \text { as } \xi \rightarrow 0 .
$$

By the variation of constant, a unique solution $H_{1}(\xi)$ of $(3.46)$ with $\cos \left(2 U_{1}\right)$ replaced by $\cos \left(2 U_{\bar{\alpha}}\right)$ is given by

$$
H_{1}(\xi)=H_{0}(\xi) \int_{0}^{\xi} \frac{d \nu}{\left\{H_{0}(\nu)\right\}^{2} \nu^{d-1}} \int_{0}^{\nu} \eta^{d-1} H_{0}(\eta) T(\eta) d \eta .
$$

Because the asymptotics of $H_{1}(\xi)$ as $\xi \rightarrow \infty$ is important in our approach, we shall carefully compute the first and the second leading terms of $T(\xi)$ and $H_{0}(\xi)$. We begin with $T(\xi)$. Recall the asymptotic expansions of $U_{\alpha}(\xi)$ (cf. (2.12c)) as $\xi \rightarrow \infty$. We may differentiate it with respect to $\xi$ and $\alpha$, respectively, whence:

$$
\begin{aligned}
H_{0}(\xi) & =h \gamma \bar{\alpha}^{-\gamma-1} \xi^{-\gamma}+O\left(\xi^{-\gamma-\omega}\right) \\
H_{0}^{\prime}(\xi) & =h \gamma \bar{\alpha}^{-\gamma-1}(-\gamma) \xi^{-\gamma-1}+O\left(\xi^{-\gamma-\omega-1}\right) \\
T(\xi) & =\frac{1}{2} h \gamma \xi^{-\gamma}+O\left(\xi^{-\gamma-\omega}\right)
\end{aligned}
$$

The asymptotics (3.49a) follows from (3.47) and (2.12d), whereas its derivative (3.49b) is shown by differentiating (3.47) in $\xi$ and using the differential equation for $U_{1}$ with asymptotics of $U_{1}$ and $U_{1}^{\prime}$. The asymptotics $(3.49 \mathrm{c})$ of $T(\xi)$ is a direct consequence of (2.12d). The asymptotics of $H_{1}(\xi)$ as $\xi \rightarrow \infty$ may be computed by substituting (3.49) in (3.48) with the help of H'Lôpital rule as follows:

$$
\begin{aligned}
& \qquad H_{1}(\xi)=C_{1} \xi^{-\gamma+2}+\left\{\begin{array}{ll}
O\left(\xi^{-\gamma-\omega+2}\right) & (\omega<2) \\
O\left(\xi^{-\gamma} \log \xi\right) & (\omega=2) \\
O\left(\xi^{-\gamma}\right) & (\omega>2)
\end{array} \text { as } \xi \rightarrow \infty\right. \\
& \text { with } C_{1}=\frac{h \gamma}{4(2+\omega)} \text {. }
\end{aligned}
$$

We just emphasize that the leading term of $H_{1}(\xi)$ does not depend on $\bar{\alpha}$. Formal differentiation of (3.50) suggests

$$
H_{1}^{\prime}(\xi)=C_{1}(-\gamma+2) \xi^{-\gamma+1}+O\left(\xi^{-\gamma-\omega+1}\right) \quad \text { as } \quad \xi \rightarrow \infty
$$

which is justified by differentiating (3.48) and using the asymptotics of the resulting functions. We are now prepared to state the most crucial results in this section. 
Lemma 3.6. Let $\theta \in(0,1)$ be the constant satisfying (3.1a). Then there exists a positive smooth function $H(\xi)$ on $(0, \infty)$ satisfying

$$
H(\xi)= \begin{cases}O\left(\xi^{k}\right) & \text { as } \xi \rightarrow 0 \\ O\left(\xi^{-\gamma}\right) & \text { as } \xi \rightarrow \infty\end{cases}
$$

such that

$$
\left|\Phi(y, s)-U_{1}\left(\frac{y}{\varepsilon(s)}\right)\right| \leq \varepsilon(s)^{2 \theta} H\left(\frac{y}{\varepsilon(s)}\right)
$$

for $y \leq \varepsilon_{0}(s)^{\theta}, s_{0} \leq s \leq s_{1}$.

Proof. Set

$$
\begin{aligned}
\Phi_{\text {out }}(y, s) & =\frac{\pi}{2}+a_{n_{0}}^{*}(s) \phi_{n_{0}}(y) ; \quad a_{n_{0}}^{*}(s)=-\frac{h}{c_{n_{0}}} \varepsilon(s)^{\gamma}, \\
\Phi_{\text {inn }}(y, s) & =U_{1}\left(\frac{y}{\varepsilon(s)}\right) .
\end{aligned}
$$

In the matching region $\varepsilon(s) \ll y \ll 1$, we obtain

$$
\Phi_{\text {out }}(y, s)-\Phi_{\text {inn }}(y, s)=a_{n_{0}}^{*}(s) y^{-\gamma}\left(\psi_{n_{0}}(y)-c_{n_{0}}\right)+O\left(\left(\frac{y}{\varepsilon(s)}\right)^{-\gamma-\delta}\right)
$$

The explicit formula of the eigenfunctions $\psi_{n}$ in Proposition 2.1 implies

$$
\psi_{n}(y)-c_{n}=c_{n}\left[\frac{(-n)}{(-\gamma+d / 2) \cdot 1 !} \frac{y^{2}}{4}+\sum_{j=2}^{n} \frac{(-n)_{j}}{(-\gamma+d / 2)_{j} \cdot j !}\left(\frac{y^{2}}{4}\right)^{j}\right]
$$

for all $n \geq 2$. Recalling the constant $C_{1}$ as in (3.50b), we obtain

$$
\left|\Phi_{\text {out }}(y, s)-\Phi_{\text {inn }}(y, s)-C_{1} \varepsilon(s)^{\gamma} y^{-\gamma+2}\right| \leq C \varepsilon(s)^{\gamma+4 \theta} y^{-\gamma}
$$

for $y=\varepsilon_{0}(s)^{\theta}$. Combining (3.54) with the assumption $\Phi \in \overline{\mathcal{A}\left(s_{0}, s_{1} ; 1\right)}$, we deduce

$$
\left|\Phi(y, s)-\Phi_{\operatorname{inn}}(y, s)-C_{1} \varepsilon(s)^{\gamma} y^{-\gamma+2}\right| \leq 2 \varepsilon(s)^{\gamma+2 \theta} y^{-\gamma}
$$

for $y=\varepsilon_{0}(s)^{\theta}, s_{0} \leq s \leq s_{1}$. By means of the inner variables, this estimate reads

$$
\varepsilon(s)^{2} H_{1}(\xi)-M_{0} \varepsilon(s)^{2 \theta} H_{0}(\xi) \leq U(\xi, s)-U_{1}(\xi) \leq \varepsilon(s)^{2} H_{1}(\xi)+M_{0} \varepsilon(s)^{2 \theta} H_{0}(\xi)
$$

with some constant $M_{0}>0$ for $\xi=\varepsilon_{0}(s)^{\theta} / \varepsilon(s), s_{0} \leq s \leq s_{1}$.

We will try to construct super- and subsolutions $W_{ \pm}(\xi, s)$ of (3.43) of the form:

$$
\begin{aligned}
V_{ \pm}(\xi, s) & :=\mu_{ \pm}(s) H_{1}(\xi) \pm M \varepsilon(s)^{2 \theta} H_{0}(\xi) . \\
\mu_{ \pm}(s) & =\varepsilon(s)^{2}\left\{1 \mp \varepsilon(s)^{\theta}\right\} .
\end{aligned}
$$


We shall first select plus sign from \pm of $(3.56 \mathrm{a})$ and prove that $V=V_{+}$is a supersolution. We now particularize the free parameter $\bar{\alpha}$ as $\bar{\alpha}=2$. A direct computation shows that

$$
\begin{aligned}
\mathcal{N} V \equiv & V_{\xi \xi}+\frac{d-1}{\xi} V_{\xi}-\frac{\Lambda_{k}}{\xi^{2}} \cos \left(2 U_{1}\right) V-\mu(s) \frac{\xi U_{1}^{\prime}}{2}-\mu(s) \frac{\xi V_{\xi}}{2}- \\
& -\frac{\Lambda_{k}}{2 \xi^{2}}\left\{\sin (2 U)-\sin \left(2 U_{1}\right)-2 \cos \left(2 U_{1}\right) V\right\}-\varepsilon(s)^{2} \frac{\partial V}{\partial s} \\
= & \frac{\Lambda_{k}}{\xi^{2}}\left[\left\{\cos \left(2 U_{2}\right)-\cos \left(2 U_{1}\right)\right\} V-\left\{\sin \left(2 U_{1}+2 V\right)-\sin \left(2 U_{1}\right)-\cos \left(2 U_{1}\right) 2 V\right\}\right]- \\
- & \mu(s) \frac{\xi}{2}\left[\mu_{+}(s) H_{1}^{\prime}+M \varepsilon(s)^{2 \theta} H_{0}^{\prime}\right]-\varepsilon(s)^{2}\left\{\dot{\mu}_{+}(s) H_{1}+M \frac{d}{d s}\left(\varepsilon(s)^{2 \theta}\right) H_{0}\right\}+ \\
+ & \left\{\mu_{+}(s)-\mu(s)\right\} T .
\end{aligned}
$$

The functions in (3.57b) and (3.57c) should be considered as source terms. For instance, we have

$$
\left|-\mu(s) \mu_{+}(s) \frac{\xi H_{1}^{\prime}(\xi)}{2}\right| \leq C \varepsilon(s)^{2+2 \theta}(1+\xi)^{-\gamma}
$$

for $\xi \leq 2 \varepsilon_{0}(s)^{\theta-1}, s_{0} \leq s \leq s_{1}$. Similar estimates for the source terms in (3.57c) may readily be obtained. On the other hand, the definition of $\mu_{+}$implies that

$$
\left\{\mu_{+}(s)-\mu(s)\right\} T(\xi) \approx-\varepsilon(s)^{2+\theta} \xi^{-\gamma} \quad \text { as } \xi \rightarrow \infty,
$$

which is larger than the quantity in (3.58) for $\xi \leq 2 \varepsilon_{0}(s)^{\theta-1}$. We thus obtain

$$
\mathcal{N} V_{+} \leq \frac{\Lambda_{k}}{\xi^{2}}\left[\left\{\cos \left(2 U_{2}\right)-\cos \left(2 U_{1}\right)\right\} V_{+}-\left\{\sin \left(2 U_{1}+2 V_{+}\right)-\sin \left(2 U_{1}\right)-\cos \left(2 U_{1}\right) 2 V_{+}\right\}\right]
$$

as long as $\xi \leq 2 \varepsilon_{0}(s)^{\theta-1}, s_{0} \leq s \leq s_{1}$. Negativity of this remaining term is verified due to our choice $\bar{\alpha}=2$. Indeed, the asymptotics of $U_{\alpha}(\xi)$ implies

$$
\begin{aligned}
U_{2}(\xi)-U_{1}(\xi) & =h\left(-2^{-\gamma}+1\right) \xi^{-\gamma}+O\left(\xi^{-\gamma-\omega}\right), \\
-\sin \left(2 U_{2}\right) & =\left(2 U_{2}-\pi\right)+O\left(\left(2 U_{2}-\pi\right)^{3}\right) \\
& =-2^{-\gamma+1} h \xi^{-\gamma}+O\left(\xi^{-\gamma-\omega}\right)+O\left(\xi^{-3 \gamma}\right) .
\end{aligned}
$$

Since $-\sin x$ is monotone increasing in $(-\pi / 2, \pi)$, we obtain

$$
\begin{aligned}
\left\{\cos \left(2 U_{2}\right)-\cos \left(2 U_{1}\right)\right\} V_{+} & \leq-\sin \left(2 U_{2}\right) \cdot 2 h\left(-2^{-\gamma}+1\right)\left[\xi^{-\gamma}+O\left(\xi^{-\gamma-\omega}\right)\right] \cdot 2 V_{+} \\
& \leq-D \varepsilon(s)^{2 \theta}\left[\xi^{-3 \gamma}+O\left(\xi^{-3 \gamma-\omega}\right)\right],
\end{aligned}
$$

where $D>0$ is a constant depending on $d, h, \gamma, M$, and $C_{0}$. On the other hand, we have

$$
\begin{aligned}
\sin \left(2 U_{1}+2 V_{+}\right)-\sin \left(2 U_{1}\right)-\cos \left(2 U_{1}\right) 2 V_{+} & =-\sin \left(2 U_{1}\right) \cdot\left(2 V_{+}\right)^{2}+O\left(\left(2 V_{+}\right)^{3}\right) \\
& =\varepsilon(s)^{4} O\left(\xi^{-3 \gamma+4}\right)+\varepsilon(s)^{4 \theta} O\left(\xi^{-3 \gamma}\right)
\end{aligned}
$$

for $1 \ll \xi \leq 2 \varepsilon(s)^{\theta-1}$. Notice that

$$
\varepsilon(s)^{4} \xi^{-3 \gamma+4} \leq C \varepsilon(s)^{2+2 \theta} \xi^{-3 \gamma+2} \leq 4 C \varepsilon(s)^{4 \theta} \xi^{-3 \gamma}
$$


for $1 \ll \xi \leq 2 \varepsilon(s)^{\theta-1}$, whence $\mathcal{N} V<0$. As for $\xi=O(1)$, negativity of $\mathcal{N} V$ in any bounded interval is verified by the presence of the term $\left\{\cos \left(2 U_{2}\right)-\cos \left(2 U_{1}\right)\right\} \cdot M \varepsilon(s)^{2 \theta} H_{0}(\xi)$. Therefore the function $V_{+}$is a supersolution for $\xi \leq 2 \varepsilon_{0}(s)^{\theta-1}, s_{0} \leq s \leq s_{1}$.

To construct a subsolution, we select minus sign from \pm of (3.56). In this case we have

$$
\left\{\mu_{-}(s)-\mu(s)\right\} T(\xi) \approx \varepsilon(s)^{2+\theta} \xi^{-\gamma} \text { for } \xi \gg 1
$$

and $\left\{\cos \left(2 U_{2}\right)-\cos \left(2 U_{1}\right)\right\} \cdot(-M) \varepsilon(s)^{2 \theta}$ dominates in any bounded interval, respectively. The detail is similar to the construction of the supersolution and is thus omitted.

We just check that the initial condition satisfies

$$
\left|\Phi_{0}(y)-U_{1}\left(\frac{y}{\varepsilon\left(s_{0}\right)}\right)-\varepsilon\left(s_{0}\right)^{2} H_{1}\left(\frac{y}{\varepsilon\left(s_{0}\right)}\right)\right| \leq C \varepsilon\left(s_{0}\right)^{2 \theta} H_{0}\left(\frac{y}{\varepsilon\left(s_{0}\right)}\right)
$$

with some constant $C>0$ for $y \leq \varepsilon\left(s_{0}\right)^{\theta}$. This is clearly satisfied for $y \leq \varepsilon\left(s_{0}\right)^{2 \theta}$, since $\Phi_{0}(y) \equiv U_{1}\left(y / \varepsilon\left(s_{0}\right)\right)$ there and $H_{1}(\xi)=O\left(\xi^{-\gamma+2}\right)$ for $\xi \gg 1$. It follows from (3.10) that

$$
\left|\Phi_{0}(y)-\Phi_{\text {out }}\left(y, s_{0}\right)\right| \leq \sum_{j=0}^{n_{0}-1}\left|\alpha_{j} \phi_{j}(y)\right|+\left|\alpha_{n_{0}}+\frac{h}{c_{n_{0}}} \phi_{n_{0}}(y)\right| \leq C \varepsilon\left(s_{0}\right)^{\gamma+2 \theta} y^{-\gamma}
$$

for $\varepsilon\left(s_{0}\right)^{2 \theta} \leq y \leq 1$. Combining this estimate with (3.50), (3.54), we obtain (3.59). Rewriting (3.59) by $\xi$ and choosing constant $M>0$ in (3.56) large enough, we conclude

$$
V_{-}\left(\xi, s_{0}\right)<U\left(\xi, s_{0}\right)-U_{1}(\xi)<V_{+}\left(\xi, s_{0}\right) \quad \text { for } \xi \leq \varepsilon\left(s_{0}\right)^{\theta-1} .
$$

Comparison principle then concludes the proof.

\subsection{A priori estimates of lower Fourier coefficients}

Let us write

$$
\Phi(y, s)=\frac{\pi}{2}+\sum_{n=0}^{n_{0}} a_{n}(s) \phi_{n}(y)+Q(y, s),
$$

where $Q(\cdot, s)$ satisfies the orthogonal condition $\left\langle Q(\cdot, s), \phi_{n}\right\rangle=0$ for $n=0, \ldots, n_{0}$.

Lemma 3.7. Assume that $2 \gamma>\omega$ holds. Suppose that $P\left(\alpha_{0}, \ldots, \alpha_{n_{0}} ; s_{1}\right)=0$ for some $\alpha \in \mathcal{U}_{s_{0}, s_{1}}$. Then:

$$
\begin{aligned}
\left|a_{n}(s)+\chi c_{n} \int_{s}^{\infty} e^{\lambda_{n}(\tau-s)} \varepsilon(\tau)^{\gamma+\omega} d \tau+\int_{s}^{s_{1}} e^{\lambda_{n}(\tau-s)} Y(\varepsilon(\tau), \Phi(\cdot, \tau)) d \tau\right| \\
\leq \begin{cases}C \varepsilon(s)^{\gamma+\omega+4 \theta} & \left(n \leq n_{0}-1\right) \\
C \varepsilon(s)^{\gamma+4 \theta} & \left(n=n_{0}\right)\end{cases}
\end{aligned}
$$

for $s_{0} \leq s \leq s$.

Proof. We first show:

$$
\left|\left\langle g(W)-g^{\prime}\left(W_{i n n}\right)\left(W-W_{i n n}\right) \chi_{\left\{|y| \leq \varepsilon(s)^{2 \theta}\right\}}, \psi_{n}\right\rangle-\chi c_{n} \varepsilon(s)^{\gamma+\omega}\right| \leq C \varepsilon(s)^{\gamma+\omega+4 \theta} .
$$


Since $g^{\prime}\left(W_{i n n}\right)=\Lambda_{k} y^{-2}\left\{\cos \left(2 y^{-\gamma} W_{i n n}\right)-1\right\}$, it follows that

$$
\left\langle g^{\prime}\left(W_{\text {inn }}\right)\left(W-W_{\text {inn }}\right) \chi_{\left\{|y| \leq \varepsilon_{0}(s)^{2 \theta}\right\}}, \psi_{n_{0}}\right\rangle=Y(\varepsilon(s), \Phi(\cdot, s))
$$

and

$$
\begin{aligned}
g(W)-g\left(W_{i n n}\right)-g^{\prime}\left(W_{i n n}\right) & \left(W-W_{i n n}\right) \\
& =\frac{\Lambda_{k}}{2} y^{\gamma-2}\left[\sin (2 v)-\sin \left(2 v_{i n n}\right)-\cos \left(2 v_{i n n}\right)\left(2 v-2 v_{i n n}\right)\right] .
\end{aligned}
$$

Lemma 3.6 implies $\left|v-v_{i n n}\right| \ll\left|v_{i n n}\right|$ for $y \leq \varepsilon(s)^{\theta}, s_{0} \leq s \leq s_{1}$, so

$$
\begin{aligned}
& \left|\int_{0}^{\varepsilon(s)^{2 \theta}}\left\{g(W)-g\left(W_{i n n}\right)-g^{\prime}\left(W_{i n n}\right)\left(W-W_{i n n}\right)\right\} \psi_{n}(y) y^{m-1} e^{-y^{2} / 4} d y\right| \\
\leq & C \int_{0}^{\varepsilon(s)^{2 \theta}} y^{\gamma-2}\left|2 U_{1}\left(\frac{y}{\varepsilon(s)}\right)-\pi\right|\left|\varepsilon(s)^{2 \theta} H\left(\frac{y}{\varepsilon(s)}\right)\right|^{2}\left|\psi_{n}(y)\right| y^{d-2 \gamma-1} \rho(y) d y \\
\leq & C \varepsilon(s)^{\gamma+\omega+4 \theta} .
\end{aligned}
$$

Finiteness of the last integral is a consequence of (3.51), that is,

$$
\left|2 U_{1}(\xi)-\pi\right||H(\xi)|^{2} \xi^{d-\gamma-3}=O\left(\xi^{d-4 \gamma-3}\right) \quad \text { as } \xi \rightarrow \infty
$$

and $d-4 \gamma-2=\omega-2 \gamma<0$. A slight modification of the argument in $\S \S 2.2$ shows

$$
\begin{aligned}
& \left|\int_{0}^{\varepsilon(s)^{2 \theta}} g\left(W_{i n n}(y, s)\right) \psi_{n}(y) y^{m-1} e^{-y^{2} / 4} d y-\chi c_{n} \varepsilon(s)^{\gamma+\omega}\right| \leq C \varepsilon(s)^{\gamma+\omega+4 \theta}, \\
& \left|\int_{\varepsilon(s)^{2 \theta}}^{\infty} g(W(y, s)) \psi_{n}(y) y^{d-2 \gamma-1} \rho(y) d y\right| \leq C \varepsilon(s)^{\gamma+\omega+(1-2 \theta)(2 \gamma-\omega)} .
\end{aligned}
$$

Since $(1-2 \theta)(2 \gamma-\omega)>4 \theta$ by (3.1a), inequality (3.61) follows from (3.62) and (3.63).

Taking inner products with $\psi_{n}$ in (3.19) and integrating the resulting ODE for $e^{\lambda_{n} s} a_{n}(s)$ over $\left[s, s_{1}\right]$, we obtain, for $n=0, \ldots, n_{0}$,

$$
\begin{aligned}
& e^{\lambda_{n} s_{1}} a_{n}\left(s_{1}\right)+\chi c_{n} \int_{s_{1}}^{\infty} e^{\lambda_{n} \tau} \varepsilon(\tau)^{\gamma+\omega} d \tau \\
& =e^{\lambda_{n} s} a_{n}(s)+\chi c_{n} \int_{s}^{\infty} e^{\lambda_{n} \tau} \varepsilon(\tau)^{\gamma+\omega} d \tau+\int_{s}^{s_{1}} e^{\lambda_{n} \tau}\left[\left\langle g(W(\cdot, \tau)), \psi_{n}\right\rangle-\chi c_{n} \varepsilon(\tau)^{\gamma+\omega}\right] d \tau .
\end{aligned}
$$

The left-hand side vanishes by assumption $P\left(\alpha ; s_{1}\right)=0$. It then follows from (3.61) that

$$
\begin{aligned}
& \left|a_{n}(s)+\chi c_{n} \int_{s}^{\infty} e^{\lambda_{n}(\tau-s)} \varepsilon(\tau)^{\gamma+\omega} d \tau+\int_{s}^{s_{1}} e^{\lambda_{n}(\tau-s)} Y(\varepsilon(\tau), \Phi(\cdot, \tau)) d \tau\right| \\
\leq & \int_{s}^{s_{1}} e^{\lambda_{n}(\tau-s)}\left|\left\langle g(W(\tau))-g^{\prime}\left(W_{i n n}\right)\left(W-W_{i n n}\right) \chi_{\left\{|y| \leq \varepsilon(s)^{2 \theta}\right\}}, \psi_{n}\right\rangle-\chi c_{n} \varepsilon(\tau)^{\gamma+\omega}\right| d \tau \\
\leq & C \int_{s}^{s_{1}} e^{\lambda_{n}(\tau-s)} \varepsilon(\tau)^{\gamma+\omega+4 \theta} d \tau \\
\leq & \begin{cases}C \varepsilon(s)^{\gamma+\omega+4 \theta} & \left(n \leq n_{0}-1\right) \\
C \varepsilon(s)^{\gamma+4 \theta} & \left(n=n_{0}\right)\end{cases}
\end{aligned}
$$

for $1 \ll s_{0} \leq s \leq s_{1}$. The proof is now complete. 
Recalling our definition of $\varepsilon(s)$ given in (3.16), we readily obtain the following corollary.

Corollary 3.8. Under the same assumptions as of Lemma 3.7, there holds

$$
\sum_{j=0}^{n_{0}-1}\left|a_{j}(s)\right|+\left|a_{n_{0}}(s)+\frac{h}{c_{n_{0}}} \varepsilon(s)^{\gamma}\right|=o\left(\varepsilon(s)^{\gamma+3 \theta}\right)
$$

for $1 \ll s_{0} \leq s \leq s_{1}$.

We then proceed to consider the case $\omega>2 \gamma$.

Lemma 3.9. Assume that $\omega>2 \gamma$ holds. Suppose that $P\left(\alpha_{0}, \ldots, \alpha_{n_{0}} ; s_{1}\right)=0$ for some $\alpha \in \mathcal{U}_{s_{0}, s_{1}}$. Then for any $\nu>0$ there exists $s_{0}$ large enough such that

$$
\left|a_{n_{0}}(s)-a_{n_{0}}^{*}(s)\right|<\nu \varepsilon(s)^{\gamma+2 \theta}
$$

for $s_{0} \leq s \leq s$, where $a_{n_{0}}^{*}(s)=-\frac{h}{c_{n_{0}}} \varepsilon(s)^{\gamma}(c f .(3.26 \mathrm{~d}))$.

Proof. We first show that there exists $\delta>0$ such that

$$
\left|\left\langle g(W)-\left(g_{L}\left(W_{\text {out }}\right)+g_{L}^{\prime}\left(W_{\text {out }}\right)\left(W-W_{\text {out }}\right)\right) \chi_{\left\{y>\varepsilon_{0}(s)^{\theta^{\prime}}\right\}}, \psi_{n_{0}}\right\rangle\right| \leq C \varepsilon(s)^{3 \gamma+(2+\delta) \theta}
$$

for $s_{0} \leq s \leq s$, where $g_{L}(W)$ and $W_{\text {out }}$ are the functions as in (3.25) and (3.26d), respectively. Computations similar to those in $\S \S 2.2$ shows that

$$
\left|\int_{0}^{\varepsilon(s)^{\theta^{\prime}}} g(W(y, s)) \psi_{n_{0}}(y) y^{m-1} \rho d y\right| \leq C \varepsilon(s)^{3 \gamma+\theta^{\prime}(\omega-2 \gamma)},
$$

where the change of variable $y=\varepsilon(s) \xi$ has been used. Since $2 \gamma=4 n_{0} \in \mathbb{N}$, the assumption $\omega>2 \gamma$ actually means $\omega-2 \gamma \geq 1$. Our basic assumption $\Phi \in \overline{\mathcal{A}\left(s_{0}, s_{1} ; 1\right)}$ implies

$$
\left|W(y, s)-W_{\text {out }}(y, s)\right| \leq C \varepsilon_{0}(s)^{\gamma+2 \theta}\left(1+y^{2 n_{0}+1}\right)
$$

for $\varepsilon_{0}(s)^{\theta} \leq y<\infty, s_{0} \leq s \leq s_{1}$. This last estimate holds also for $\varepsilon(s)^{\theta^{\prime}} \leq y \leq \varepsilon_{0}(s)^{\theta}$, $s_{0} \leq s \leq s_{1}$, due to Lemma 3.6 and $(2.12 \mathrm{c})$. We then readily see that

$$
\begin{aligned}
\mid\left\langle\left(g_{L}(W)-g_{L}\left(W_{\text {out }}\right)-g_{L}^{\prime}\left(W_{\text {out }}\right)\left(W-W_{\text {out }}\right)\right)\right. & \left.\chi_{\left\{y>\varepsilon_{0}(s)^{\theta^{\prime}}\right\}}, \psi_{n_{0}}\right\rangle \mid \\
& \leq C \varepsilon(s)^{3 \gamma+4 \theta} \int_{0}^{\infty}\left|\psi_{n_{0}}(y)\right| y^{m-2 \gamma-3} \rho d y
\end{aligned}
$$

and the last integral converges since $m-2 \gamma-3=\omega-2 \gamma-1>-1$ by assumption. The error arising in replacing $g(W)$ by its leading term $g_{L}(W)$ in $\left\{y>\varepsilon_{0}(s)^{\theta^{\prime}}\right\}$ is readily estimated by Taylor as well as the exponential weight function $\rho$. The result is:

$$
\left|\int_{\varepsilon_{0}(s)^{\theta^{\prime}}}^{\infty}\left\{g(W)-g_{L}(W)\right\} \psi_{n_{0}}(y) y^{m-1} \rho d y\right| \leq C \varepsilon(s)^{3 \gamma+2\left(1-\theta^{\prime}\right) \gamma}
$$

and $2\left(1-\theta^{\prime}\right) \gamma>2 \theta^{\prime}>4 \theta$. Summarizing these estimates, we obtain (3.66). 
Arguing as in the proof of Lemma 3.7, we obtain

$$
\begin{aligned}
& a_{n_{0}}\left(s_{1}\right)-\chi \int_{s_{1}}^{\infty}\left\{a_{n_{0}}^{*}(\tau)\right\}^{3} d \tau \\
= & a_{n_{0}}(s)-\chi \int_{s}^{\infty}\left\{a_{n_{0}}^{*}(\tau)\right\}^{3} d \tau-\int_{s}^{s_{1}}\left\langle g_{L}^{\prime}\left(W_{\text {out }}\right)\left(W-W_{\text {out }}\right) \chi_{\left\{y>\varepsilon_{0}(s)^{\theta^{\prime}}\right\}}, \psi_{n_{0}}\right\rangle d \tau \\
& +\int_{s}^{s_{1}}\left\langle g(W)-\left(g_{L}\left(W_{\text {out }}\right)+g_{L}^{\prime}\left(W_{\text {out }}\right)\left(W-W_{\text {out }}\right)\right) \chi_{\left\{y>\varepsilon_{0}(s)^{\theta^{\prime}}\right\}}, \psi_{n_{0}}\right\rangle d \tau .
\end{aligned}
$$

The assumption $P\left(\alpha ; s_{1}\right)=0$ means that the left-hand side vanishes. Due to the definition of $\varepsilon(s)$ given in (3.26a), we then obtain, by (3.66),

$$
\begin{aligned}
\left|a_{n_{0}}(s)-a_{n_{0}}^{*}(s)\right| & =\left|\int_{s}^{s_{1}}\left\langle g(W)-\left(g_{L}\left(W_{\text {out }}\right)+g_{L}^{\prime}\left(W_{\text {out }}\right)\left(W-W_{\text {out }}\right)\right) \chi_{\left\{y>\varepsilon_{0}(s)^{\theta^{\prime}}\right\}}, \psi_{n_{0}}\right\rangle d \tau\right| \\
& \leq C \varepsilon(s)^{\gamma+(2+\delta) \theta} .
\end{aligned}
$$

The proof is now complete.

\subsection{Pointwise bounds for higher Fourier modes}

We estimate the higher mode:

$$
E(y, s)=W(y, s)-\sum_{j=0}^{n_{0}} a_{j}(s) \psi_{j}(y), \quad\left\langle E(\cdot, s), \psi_{j}\right\rangle=0 \quad \text { for } j=0, \ldots, n_{0},
$$

which solves

$$
\begin{gathered}
E_{s}=\Delta_{y} E-\frac{y \cdot \nabla_{y} E}{2}+\frac{\gamma}{2} E+R, \\
R=R(y, s)=g(W(y, s))-\sum_{j=0}^{n_{0}}\left\langle g(W(\cdot, s)), \psi_{j}\right\rangle \psi_{j}(y) .
\end{gathered}
$$

Here and henceforth, we abuse the notation of $y$ to denote the variable in $\mathbb{R}^{m}$ with $m=d-2 \gamma$ and the counterparts of the partial derivatives are denoted by $\nabla_{y}$ and $\Delta_{y}$, respectively. The standard semigroup theory shows

$$
E_{s}=T\left(s-s_{0}\right) E_{0}+\int_{s_{0}}^{s} T(s-\tau) R(\cdot, \tau) d \tau, \quad s>s_{0},
$$

with $E_{0}=E\left(\cdot, s_{0}\right)$ and

$$
\begin{aligned}
{[T(s) E](y) } & =\frac{e^{(\gamma / 2) s}}{\left\{4 \pi\left(1-e^{-s}\right)\right\}^{m / 2}} \int_{\mathbb{R}^{m}} \exp \left(-\frac{\left|y e^{-s / 2}-z\right|^{2}}{4\left(1-e^{-s}\right)}\right) E(z) d z \\
& =\sum_{j=0}^{\infty} e^{-\lambda_{j} s}\left\langle E, \psi_{j}\right\rangle \psi_{j}(y)
\end{aligned}
$$

for $s>0$. The convergence of the series are understood in the norm of $L_{\rho}^{2}\left(\mathbb{R}^{m}\right)$ as well as the uniform topology in every compact subset of $\mathbb{R}^{m}$. This last statement is due to uniform boundedness of the Laguerre polynomials $\psi_{j}(j=0,1 \ldots)$. 
Our goal in this subsection is to show: If $\Phi \in \overline{\mathcal{A}\left(s_{0}, s_{1} ; 1\right)}$, then there holds

$$
|E(y, s)| \ll \varepsilon(s)^{\gamma+2 \theta}\left(1+y^{2 n_{0}+1}\right), \quad \varepsilon_{0}(s)^{\theta} \leq|y|<\infty, s_{0} \leq s \leq s_{1} .
$$

To this end, we first show (3.69) for short-time $s_{0} \leq s \leq s_{0}+1$ and then extend it to long-time $s_{0}+1<s$, to get (3.69) for the whole time interval $s_{0} \leq s \leq s_{1}$. In the following, we always assume $\Phi \in \overline{\mathcal{A}\left(s_{0}, s_{1} ; 1\right)}$, even if it is not stated explicitly, and the indicator function of interval $I$ is denoted by $\chi(I)(\cdot)$

\subsubsection{A priori estimates for short-time interval}

Lemma 3.10. For any $\nu>0$ there exists $s_{0}$ large enough such that

$$
\left|\left[T\left(s-s_{0}\right) E_{0}\right](y)\right|<\nu \varepsilon(s)^{\gamma+2 \theta}\left(1+|y|^{2 n_{0}+1}\right)
$$

for $\varepsilon(s)^{\theta} \leq|y|<\infty, s_{0} \leq s \leq s_{0}+1$.

Proof. We will estimate the left-hand side of (3.70) by (3.68a). Recall (3.15):

$$
\left|E_{0}(y)\right| \leq \begin{cases}C \varepsilon_{0}\left(s_{0}\right)^{\gamma} & \text { for }|y| \leq \varepsilon_{0}\left(s_{0}\right)^{\theta} \\ C \varepsilon_{0}\left(s_{0}\right)^{\gamma+4 \theta}\left(1+|y|^{2 n_{0}+1 / 2}\right) & \text { for } \varepsilon_{0}\left(s_{0}\right)^{\theta}<|y|<\infty\end{cases}
$$

The former estimate of (3.71) implies

$$
\left|\left[T\left(s-s_{0}\right) E_{0} \chi\left(\left[0, \varepsilon_{0}\left(s_{0}\right)^{2 \theta}\right]\right)\right](y)\right| \leq C \varepsilon\left(s_{0}\right)^{\gamma+m \theta}\left(1+|y|^{2 n_{0}+1 / 2}\right)
$$

for $\varepsilon_{0}(s)^{\theta} \leq|y|$, since $\sup _{x>0} x^{m / 2} e^{-x / 16}<\infty$. It follows from the latter estimate of (3.71) that

$$
\left|\left[T\left(s-s_{0}\right) E_{0} \chi\left(\left[\varepsilon_{0}\left(s_{0}\right)^{2 \theta}, 2 \eta\left(s_{0}\right)\right]\right)\right](y)\right| \leq C \varepsilon\left(s_{0}\right)^{\gamma+4 \theta}\left(1+|y|^{2 n_{0}+1 / 2}\right)
$$

for all $y \in \mathbb{R}^{m}$. As long as the region $\left\{|y|<\eta\left(s_{0}\right)\right\}$ is concerned, there holds ||$y|-| z|| \geq$ $|z| / 2$ for $|z|>2 \eta\left(s_{0}\right)$, and hence

$$
\left|\left[T\left(s-s_{0}\right) E_{0} \chi\left(\left(2 \eta\left(s_{0}\right), \infty\right)\right)\right](y)\right| \leq C \varepsilon\left(s_{0}\right)^{\gamma} \exp \left(-\frac{\eta(s)^{2}}{8}\right),
$$

whence the desired bound.

We finally consider the region $\left\{|y|>\eta\left(s_{0}\right)\right\}$. Split the integral as

$$
\int_{\left\{|z|>2 \eta\left(s_{0}\right)\right\}}=\int_{\{|z|>4|y|\}}+\int_{\left\{2 \eta\left(s_{0}\right) \leq|z| \leq|y| / 4\right\}} .
$$

The former integral may be estimated as in the previous one. On the other hand, in the region of the latter, we have $|z|^{2 n_{0}+1 / 2} \leq 2^{-1 / 2} \varepsilon_{0}\left(s_{0}\right)^{(\gamma+2 \theta) / 3}|z|^{2 n_{0}+1}$, whence:

$$
\left|\left[T\left(s-s_{0}\right) E_{0} \chi\left(\left[2 \eta\left(s_{0}\right),|y| / 4\right]\right)\right](y)\right| \leq C \varepsilon\left(s_{0}\right)^{\gamma+(\gamma+2 \theta) / 3}\left(1+|y|^{2 n_{0}+1}\right)
$$

for $s_{0} \leq s \leq s_{0}+1$. Putting these estimates together, we obtain the desired estimate (3.70). The proof is complete. 
Lemma 3.11. For any $\nu>0$ there exists $s_{0}$ large enough such that

$$
\left|\int_{s_{0}}^{s}[T(s-\tau) R(\cdot, \tau)](y) d \tau\right|<\nu \varepsilon(s)^{\gamma+2 \theta}\left(1+|y|^{2 n_{0}+1}\right)
$$

for $\varepsilon_{0}(s)^{\theta} \leq|y|<\infty, s_{0} \leq s \leq s_{0}+1$.

Proof. Note that

$$
\int_{s_{0}}^{s} T(s-\tau) R(\cdot, \tau) d \tau=\int_{s_{0}}^{s} T(s-\tau) g(W) d \tau-\sum_{j=0}^{n_{0}} \int_{s_{0}}^{s} T(s-\tau)\left\langle g(W), \psi_{j}\right\rangle \psi_{j} d \tau .
$$

The second term is easily estimated, since $\left|\left\langle g(W), \psi_{j}\right\rangle\right| \leq C \varepsilon(s)^{\gamma+\min \{\omega, 2 \gamma\}}$. To estimate the first term, let us write

$$
g(W)=g(W) \chi_{\left\{|y| \leq \varepsilon(s)^{\theta} / 8\right\}}+g(W) \chi_{\left\{|y|>\varepsilon(s)^{\theta} / 8\right\}}=: h_{1}(y, s)+h_{2}(y, s) .
$$

Consider the term involving $h_{1}$. By the change of variable $z=\varepsilon(\tau) \zeta$, one has

$$
\begin{aligned}
& \int_{\left\{|z| \leq \varepsilon(\tau)^{\theta / 8\}}\right.} \exp \left(-\frac{\left|y e^{-(s-\tau)}-z\right|^{2}}{4\left(1-e^{-(s-\tau)}\right)}\right)\left|g\left(W_{i n n}\right)\right| d z \\
\leq & C \varepsilon(\tau)^{m+\gamma-2} \int_{B} \exp \left(-\frac{\left|y e^{-(s-\tau)}-\varepsilon(\tau) \zeta\right|^{2}}{4\left(1-e^{-(s-\tau)}\right)}\right)|\zeta|^{\gamma-2}\left|\sin \left(2 U_{1}-\pi\right)-\left(2 U_{1}-\pi\right)\right| d \zeta,
\end{aligned}
$$

where $B=\left\{\zeta \in \mathbb{R}^{m}:|\zeta| \leq \varepsilon(\tau)^{-(1-\theta)} / 8\right\}$ and $U_{1}=U_{1}(|\zeta|)$. We want to replace $\varepsilon(\tau) \zeta$ by 0 in the exponential factor. To estimate the error that may arise, we take advantage of an elementary inequality

$$
\left|e^{-(w-v)^{2}}-e^{-w^{2}}\right| \leq C e^{-w^{2} / 2}|w||v| \quad \text { provided that } \quad|v| \leq \frac{1}{2}|w|
$$

with

$$
v=\frac{\varepsilon(\tau) \zeta}{2 \sqrt{1-e^{-(s-\tau)}}}, \quad w=\frac{y e^{-(s-\tau) / 2}}{2 \sqrt{1-e^{-(s-\tau)}}}
$$

As a result, we obtain

$$
\begin{array}{rl} 
& \int_{\left\{|z| \leq \varepsilon(\tau)^{\theta} / 8\right\}} \exp \left(-\frac{\left|y e^{-(s-\tau)}-z\right|^{2}}{4\left(1-e^{-(s-\tau)}\right)}\right)\left|g\left(W_{i n n}\right)\right| d z \\
\leq C & C(\tau)^{m+\gamma-2}\left\{\exp \left(-\frac{\left|y e^{-(s-\tau)}\right|^{2}}{4\left(1-e^{-(s-\tau)}\right)}\right)+\exp \left(-\frac{\left|y e^{-(s-\tau)}\right|^{2}}{8\left(1-e^{-(s-\tau)}\right)}\right) \frac{\varepsilon(\tau)^{2 \theta}|y| e^{-(s-\tau)}}{1-e^{-(s-\tau)}}\right\} . \\
& \cdot \int_{\left\{|\zeta| \leq \varepsilon(\tau)^{\theta-1}\right\}}|\zeta|^{\gamma-2}\left|\sin \left(2 U_{1}(|\zeta|)-\pi\right)-\left(2 U_{1}(|\zeta|)-\pi\right)\right| d \zeta
\end{array}
$$

for $\varepsilon(s)^{2 \theta} \leq|y|, s_{0} \leq s \leq s_{0}+1$. Since

$$
\left|\sin \left(2 U_{1}(r)-\pi\right)-\left(2 U_{1}(r)-\pi\right)\right| \sim \frac{1}{3 !}\left|2 U_{1}(r)-\pi\right|^{3}=O\left(r^{-3 \gamma}\right)
$$


as $r \rightarrow \infty$, and $\gamma-2-3 \gamma+m-1=\omega-2 \gamma-1$, we obtain

$$
\begin{aligned}
& \int_{s_{0}}^{s} \frac{1}{\left\{1-e^{-(s-\tau)}\right\}^{m / 2}} \int_{\left\{|z| \leq \varepsilon(\tau)^{\theta / 8\}}\right.} \exp \left(-\frac{\left|y e^{-(s-\tau) / 2}-z\right|^{2}}{4\left(1-e^{-(s-\tau)}\right)}\right)\left|g\left(W_{\mathrm{inn}}\right)\right| d z d \tau \\
\leq & C \int_{s_{0}}^{s} \frac{\varepsilon(\tau)^{\gamma+\omega}\left(1+\varepsilon(\tau)^{(\theta-1)(\omega-2 \gamma)}\right)}{\left\{1-e^{-(s-\tau)}\right\}^{m / 2}} \exp \left(-\frac{\left|y e^{-(s-\tau) / 2}\right|^{2}}{4\left(1-e^{-(s-\tau)}\right)}\right) d \tau \\
\leq & C \varepsilon\left(s_{0}\right)^{\gamma+\omega}\left(1+\varepsilon\left(s_{0}\right)^{(\theta-1)(\omega-2 \gamma)}\right)|y|^{-\omega}
\end{aligned}
$$

where the change of variable $t=|y|^{2} / 4\left(1-e^{-(s-\tau)}\right)$ has bee used as well. Since $|g(W)| \leq$ $2 g\left(W_{\text {inn }}\right)$ due to Lemma 3.6, we obtain

$$
\left|\int_{s_{0}}^{s}\left[T(s-\tau) h_{1}(\cdot, \tau)\right](y) d \tau\right|<C \varepsilon(s)^{\gamma+(1-\theta) \delta}
$$

for $\varepsilon_{0}(s)^{\theta} \leq|y|<\infty$, where $\delta=\min \{2 \gamma, \omega\}$.

We shall turn our attention to the term involving $h_{2}(y, \tau)$. Recall the function $\eta(s)=$ $\left\{\varepsilon_{0}(s)\right\}^{-(2 / 3)(\gamma+2 \theta)}$. The function $g(W)$ may be estimated by a cubic function of $|y|^{-\gamma} W$ in $\left\{8^{-1} \varepsilon_{0}(s)^{\theta} \leq|y| \leq \eta(s)\right\}$ due to the assumption $\Phi \in \overline{\mathcal{A}\left(s_{0}, s_{1} ; 1\right)}$ and Lemma 3.6. We then obtain

$$
\begin{aligned}
\int_{s_{0}}^{s}\left|\left[T(s-\tau) h_{2} \chi\left(\left[\varepsilon(\tau)^{\theta} / 8, \eta(\tau)\right]\right)\right](y)\right| d \tau & \leq C \int_{s_{0}}^{s} \varepsilon(\tau)^{\gamma+2(1-\theta) \gamma-2 \theta} d \tau\left(1+|y|^{2 n_{0}+1}\right) \\
& \ll \varepsilon(s)^{\gamma+2 \theta}\left(1+|y|^{2 n_{0}+1}\right)
\end{aligned}
$$

for $\varepsilon_{0}(s)^{\theta} \leq|y|<\infty$, since $2(1-\theta) \gamma-2 \theta>2 \theta$ (cf. (3.1)). Our next task is to show:

$$
\int_{s_{0}}^{s}\left|\left[T(s-\tau) h_{2} \chi([\eta(\tau), \infty))\right](y)\right| d \tau<\nu \varepsilon(s)^{\gamma+2 \theta}\left(1+|y|^{2 n_{0}+1}\right)
$$

for $\varepsilon_{0}(s)^{\theta} \leq|y|<\infty$. Consider first the region $\{|y| \leq \eta(s) / 4\}$, in which $|z|-|y|>(3 / 4)|z|$ for any $|z|>\eta(s)$, whence:

$$
\exp \left(-\frac{\left|y e^{-(s-\tau) / 2}-z\right|^{2}}{4\left(1-e^{-(s-\tau)}\right)}\right) \leq \exp \left(-\frac{9|z|^{2}}{32\left(1-e^{-(s-\tau)}\right)}\right) \exp \left(-\frac{9 \eta(s)^{2}}{32}\right)
$$

The desired estimate then follows at once. Consider next the region $\{|y|>\eta(s) / 4\}$. Split the integral as

$$
\int_{\{|z| \geq \eta(\tau)\}}=\int_{\{\eta(\tau) \leq|z| \leq 4|y|\}}+\int_{\{4|y|<|z|\}} .
$$

The latter part may be estimated as above. As for the former, we have $|g(W(z, \tau))| \leq$ $C|z|^{\gamma-2} \leq C|z|^{2 n_{0}+1}\{\eta(\tau)\}^{-3}$ in the region of integration. Hence (3.76) holds. Summarizing, we have obtained

$$
\left|\int_{s_{0}}^{s}\left[T(s-\tau) h_{2}(\cdot, \tau)\right](y) d \tau\right|<\nu^{2} \varepsilon(s)^{\gamma+2 \theta}\left(1+|y|^{2 n_{0}+1}\right)
$$

for $\varepsilon_{0}(s)^{\theta} \leq|y|<\infty, s_{0} \leq s \leq s_{0}+1$. Combining (3.74) with (3.77), we conclude the desired estimate. The proof is complete. 


\subsubsection{A priori estimates for long-time interval}

We extend the estimates having been obtained in $\S \S \S 3.5 .1$ to the case $s_{1}>s_{0}+1$.

Lemma 3.12. For any $\nu>0$ there exists $s_{0}$ large enough such that

$$
\left|\left[T\left(s-s_{0}\right) E_{0}\right](y)\right|<\nu \varepsilon(s)^{\gamma+2 \theta}\left(1+|y|^{2 n_{0}+1}\right)
$$

for $\varepsilon_{0}(s)^{\theta} \leq|y|<\infty, s_{0}+1<s \leq s_{1}$.

Proof. Let $K>1$ be a constant to be specified later. We first show $(3.78)$ in $\left[\varepsilon_{0}(s)^{\theta}, K\right] \times$ $\left(s_{0}+1, s_{1}\right]$. The series expression (3.68b) of the semigroup $T(s)$ yields

$$
\left|\left[T\left(s-s_{0}\right) E_{0}\right](y)\right| \leq C_{K} \sum_{j=n_{0}+1}^{\infty} e^{-\lambda_{j}\left(s-s_{0}\right)}\left|\left\langle E_{0}, \psi_{j}\right\rangle\right| .
$$

Due to (3.14), we have $\left|\left\langle E_{0}, \psi_{j}\right\rangle\right| \leq C \varepsilon_{0}\left(s_{0}\right)^{\gamma+m \theta}$ for some constant $C>0$ independent of $j$ and $s_{0}$. Hence we obtain

$$
\begin{aligned}
\left|\left[T\left(s-s_{0}\right) E_{0}\right](y)\right| & \leq C_{K}^{\prime} \varepsilon(s)^{\gamma+m \theta}\left(\frac{\varepsilon\left(s_{0}\right)}{\varepsilon(s)}\right)^{\gamma+m \theta} e^{-\left(s-s_{0}\right)} \sum_{j=n_{0}+1}^{\infty} e^{-\left(\lambda_{j}-\lambda_{n_{0}+1}\right)\left(s-s_{0}\right)} \\
& \leq C_{K}^{\prime \prime} \varepsilon(s)^{\gamma+m \theta} e^{-\left(s-s_{0}\right) / 4}
\end{aligned}
$$

for $|y| \leq K, s_{0}+1<s \leq s_{1}$. In particular, (3.78) holds there.

We will extend this bound to unbounded region $[K, \infty)$. Consider the function

$$
w(y, s)=e^{-(1 / 4)\left(s-s_{0}\right)}|y|^{2 n_{0}+1} .
$$

A simple computation shows that

$$
w_{s}-\Delta w+\frac{y \cdot \nabla w}{2}-\frac{\gamma}{2} w=\frac{1}{4} e^{-(1 / 4)\left(s-s_{0}\right)}\left[|y|^{2 n_{0}+1}-K^{2}|y|^{2 n_{0}-1}\right],
$$

which is positive if $|y|^{2}>4\left(2 n_{0}+1\right)\left(2 n_{0}+m-1\right)=: K^{2}$. Namely, $w$ is a supersolution of equation $v_{s}=\Delta v-2^{-1}(y \cdot \nabla v)+(\gamma / 2) v$ in $(K, \infty) \times\left(s_{0}, s_{1}\right)$. Moreover, we have

$$
\left|E_{0}(y)\right|<\nu^{2} \varepsilon\left(s_{0}\right)^{\gamma+2 \theta}|y|^{2 n_{0}+1}
$$

for $K \leq|y|<\infty$. Furthermore, (3.80) and the corresponding short-time estimate imply

$$
\left|\left[T\left(s-s_{0}\right) E_{0}\right](y)\right|<\nu^{2} \varepsilon\left(s_{0}\right)^{\gamma+2 \theta} e^{-(1 / 4)\left(s-s_{0}\right)}|y|^{2 n_{0}+1}
$$

whenever $|y|=K, s_{0} \leq s \leq s_{1}$. We may now apply comparison principle, to get

$$
\begin{aligned}
\left|\left[T\left(s-s_{0}\right) E_{0}\right](y)\right| & \leq \nu^{2} \varepsilon\left(s_{0}\right)^{\gamma+2 \theta} w(y, s)=\nu^{2} \varepsilon(s)^{\gamma+2 \theta}\left(\frac{\varepsilon\left(s_{0}\right)}{\varepsilon(s)}\right)^{\gamma+2 \theta} e^{-(1 / 4)\left(s-s_{0}\right)}|y|^{2 n_{0}+1} \\
& <\nu \varepsilon(s)^{\gamma+2 \theta}|y|^{2 n_{0}+1}
\end{aligned}
$$

for $K<|y|, s_{0} \leq s \leq s_{1}$, which completes the proof. 
Lemma 3.13. For any $\nu>0$ there exists $s_{0}$ large enough such that

$$
\left|\int_{s_{0}}^{s}[T(s-\tau) R(\cdot, \tau)](y) d \tau\right|<\nu \varepsilon_{0}(s)^{\gamma+2 \theta}\left(1+|y|^{2 n_{0}+1}\right)
$$

for $\varepsilon_{0}(s)^{\theta} \leq|y|<\infty, s_{0}+1 \leq s \leq s_{1}$.

Proof. The proof is almost the same as of Lemma 3.12, so we state only the main points. We first notice that $\int_{s_{0}}^{s}$ in (3.84) may be replaced by $\int_{s_{0}}^{s-1}$, since the term corresponding to $\int_{s-1}^{s}$ may be estimated exactly as in the short-time estimates in $§ \S 3.5 .1$. Notice that

$$
\left|\left\langle R(\cdot, \tau), \psi_{j}\right\rangle\right| \leq C \varepsilon(\tau)^{\gamma+\omega}, \quad j=0,1, \ldots
$$

Pick $\tau \in\left[s_{0}, s-1\right]$. Since $\left\langle R(\cdot, s), \psi_{j}\right\rangle=0$ for $j=0, \ldots, n_{0}$, the series expression (3.68b) of the semigroup $T(s)$ as well as local uniform bounds of Laguerre polynomials guarantee

$$
|[T(s-\tau) R(\cdot, \tau)](y)| \leq C_{K} \varepsilon(\tau)^{\gamma+\omega} e^{-\lambda_{n_{0}+1}(s-\tau)}
$$

if $|y| \leq K$. We now let $\tau$ vary over $\left[s_{0}, s-1\right]$ and integrate $T(s-\tau) R(\cdot, \tau)$ there, to get

$$
\left|\int_{s_{0}}^{s-1}[T(s-\tau) R(\cdot, \tau)](y) d \tau\right| \leq C C_{K} \varepsilon(s)^{\gamma+\omega} e^{-(1 / 4)\left(s-s_{0}\right)}
$$

for $|y| \leq K, s_{0}+1<s \leq s_{1}$. Notice that

$$
|g(W(y, s))| \leq \begin{cases}\varepsilon(s)^{\gamma+3 \theta}|y|^{\gamma+1} & (1 \leq|y| \leq 2 \eta(s)) \\ C \varepsilon(s)^{2(\gamma+2 \theta)}|y|^{\gamma+1} & (2 \eta(s)<|y|<\infty)\end{cases}
$$

whence:

$$
|R(y, \tau)|<\nu \varepsilon(\tau)^{\gamma+2 \theta}|y|^{2 n_{0}+1}
$$

for all $|y|>K$. Comparison principle as well as integration in $\tau$ then implies

$$
\begin{aligned}
\left|\int_{s_{0}}^{s-1}[T(s-\tau) R(\cdot, \tau)](y) d \tau\right| & \leq C \nu^{2}|y|^{2 n_{0}+1} \int_{s_{0}}^{s-1} \varepsilon(\tau)^{\gamma+2 \theta} e^{-(1 / 4)(s-\tau)} d \tau \\
& <\nu \varepsilon(s)^{\gamma+2 \theta}|y|^{2 n_{0}+1}
\end{aligned}
$$

for $|y|>K, s_{0}+1<s \leq s_{1}$. The proof is now complete.

Completion of the proof of Lemma 3.5. Due to Lemmas 3.10-3.13, we conclude the estimate (3.69) on the higher Fourier mode. The a priori estimate $\Phi \in \mathcal{A}\left(s_{0}, s_{1} ; 1 / 2\right)$ then follows from Lemma 3.6, Corollary 3.8, and (3.69). The proof is complete.

\subsection{Asymptotic behavior of energy density}

Having proved theorems 3.1 and 1.1, we turn our attention to the proof of Theorem 1.5.

Proposition 3.14. Assume the same hypotheses as in Theorem 1.1. Let $u$ and $\varepsilon(s)$ be as in Theorem 1.5. Then for every $K>0$ there exists a constant $C>0$ such that

$$
\sum_{j=0}^{2} \sup _{\xi \leq K}\left|\frac{\partial^{j}}{\partial \xi^{j}}\left(u(\xi \varepsilon(s) \sqrt{T-t}, t)-U_{1}(\xi)\right)\right| \leq C \varepsilon_{0}(s)^{2 \theta}
$$

for $T / 2<t<T$. 
Proof. We appeal to classical parabolic estimates after performing suitable change of variables. Without loss of generality, we may assume that $K=1 / 4$. Recall the notations $\xi, U(\xi, s)$ used in $\S \S 3.3$ (cf. (3.43)). Let us write

$$
V(\xi, s)=U(\xi, s)-U_{1}(\xi)=\xi^{k} \tilde{Z}(\xi, \tau), \quad \tau=\int_{s_{0}}^{s} \frac{\mathrm{ds}}{\varepsilon(s)^{2}} .
$$

By direct computations, it turns out that $\tilde{Z}$ satisfies the radial version of a $(2 k+d)$ dimensional parabolic equation with uniformly bounded continuous coefficients for $|\xi|<1$, $\tau>0$. To avoid ambiguity, we shall introduce different notations: $Z(\eta, \tau)=\tilde{Z}(\xi, \tau)$, $\eta \in \mathbb{R}^{2 k+d},|\eta|=\xi$. The equation for $\tilde{Z}(\xi, \tau)$ is then recast as

$$
\begin{gathered}
Z_{\tau}=\Delta_{\eta} Z-\frac{\mu_{1}(\tau)}{2} \eta \cdot \nabla_{\eta} Z+\left[\frac{\Lambda_{k}}{|\eta|^{2}}\left(1-\cos \left(2 U_{1}\right)\right)-\frac{k \mu_{1}(\tau)}{2}\right] Z+F(\eta, \tau), \\
F(\eta, \tau)=-\mu_{1}(\tau) \frac{U_{1}^{\prime}}{2|\eta|^{k-1}}-\frac{\Lambda_{k}}{2|\eta|^{2+k}}\left[\sin \left(2 U_{1}+2|\eta|^{k} Z\right)-\sin \left(2 U_{1}\right)-\cos \left(2 U_{1}\right) 2|\eta|^{k} Z\right],
\end{gathered}
$$

where $\mu_{1}(\tau)=\mu(s)=\varepsilon(s)^{2}-2 \varepsilon(s) \dot{\varepsilon}(s), U_{1}=U_{1}(|\eta|), U_{1}^{\prime}=U_{1}^{\prime}(|\eta|)$, and $\nabla_{\eta}, \Delta_{\eta}$ denote the counterparts of differential operators in $\eta$, respectively. Fix $\tau^{*}>0$ and set $Q=$ $B_{1} \times\left(\tau^{*}, \tau^{*}+1\right]$. Due to (3.4), there is a constant $C>0$ independent of $\tau^{*}$ such that

$$
\|Z\|_{L^{\infty}(Q)} \leq C \varepsilon_{1}\left(\tau^{*}\right)^{2 \theta},
$$

where $\varepsilon_{1}(\tau)=\varepsilon_{0}(s)$, and thus $\|Z\|_{L^{p}(Q)} \leq C \varepsilon_{1}\left(\tau^{*}\right)^{2 \theta}$ for every $p>2 k+d+1$. By Talyor expansion and Proposition 2.3, we readily obtain an $L^{\infty}$-estimate on the forcing term of the form $\|F\|_{L^{\infty}(Q)} \leq C^{\prime} \varepsilon_{1}\left(\tau^{*}\right)^{2 \theta}$. Set $Q^{\prime}=B_{1 / 2} \times\left(\tau^{*}+1 / 2, \tau^{*}+1\right]$. $L^{p}$ theory for linear parabolic equations implies

$$
\|Z\|_{W_{p}^{2,1}\left(Q^{\prime}\right)} \leq D\left(\|Z\|_{L^{p}(Q)}+\|F\|_{L^{p}(Q)}\right) \leq K\left(C+C^{\prime}\right) \varepsilon_{1}\left(\tau^{*}\right)^{2 \theta},
$$

where $D>0$ is a constant depending only on $k, d$, and the parabolic distance between $Q^{\prime}$ and the parabolic boundary of $Q$. For $\lambda \in(0,1-(2 k+d+1) / p)$, let us denote by $C^{\lambda, \lambda / 2}\left(\overline{Q^{\prime}}\right)$ the standard Hölder spaces of order $\lambda$ in $\overline{Q^{\prime}}$ with respect to parabolic distance. Due to a version of Sobolev inequalities [1, Lemma 4.28, IV] as well as Taylor expansion and Proposition 2.3, estimate (3.89) implies

$$
\|F\|_{C^{\lambda, \lambda / 2}\left(\overline{Q^{\prime}}\right)} \leq C^{\prime \prime} \varepsilon_{1}\left(\tau^{*}\right)^{2 \theta},
$$

where $C^{\prime \prime}>0$ is a constant depending on $C, C^{\prime}, D, p, k, d$ but not on $\tau^{*}$. Taking account of the uniform Hölder estimates for $\dot{\varepsilon}(s)$ guaranteed by Proposition 3.3, we may verify uniform bounds of Hölder norms (independent of $\tau^{*}$ ) on coefficients of the linear part of (3.87). Hence we may apply Schauder theory. Set $Q^{\prime \prime}=B_{1 / 4} \times\left(\tau^{*}+3 / 4, \tau^{*}+1\right]$. Since $\varepsilon\left(\tau^{*}\right) \leq 2 \varepsilon(\tau)$, it follows from (3.88)-(3.90) that

$$
\|Z\|_{C^{2+\lambda, 1+\lambda / 2}\left(\overline{Q^{\prime \prime}}\right)} \leq M\left(\|Z\|_{L^{\infty}\left(Q^{\prime}\right)}+\|F\|_{C^{\lambda, \lambda / 2}\left(\overline{Q^{\prime}}\right)}\right) \leq 2 M\left(C+C^{\prime \prime}\right) \varepsilon_{1}(\tau)^{2 \theta}
$$

for $\tau^{*}+3 / 4<\tau<\tau^{*}+1$. We now let $\tau^{*}$ vary on $(0, \infty)$, to get, in particular,

$$
\|Z(\cdot, \tau)\|_{C^{2+\lambda}\left(\overline{B_{1 / 4}}\right)} \leq 2 M\left(C+C^{\prime \prime}\right) \varepsilon_{1}(\tau)^{2 \theta}, \quad \tau>1 .
$$


Notice that $V(\xi, s)=|\eta|^{k} Z(\eta, \tau)$ satisfies

$$
\begin{aligned}
\left|\frac{\partial V}{\partial \xi}\right| & \leq k|\eta|^{k-1}|Z(\eta, \tau)|+|\eta|^{k}\left|\nabla_{\eta} Z(\eta, \tau)\right| \\
\left|\frac{\partial^{2} V}{\partial \xi^{2}}\right| & \leq k(k-1)|\eta|^{k-2}|Z(\eta, \tau)|+(d-1)|\eta|^{k-1}\left|\nabla_{\eta} Z(\eta, \tau)\right|+|\eta|^{k}\left|\Delta_{\eta} Z(\eta, \tau)\right| .
\end{aligned}
$$

Estimating the right-hand sides by (3.91), we obtain (3.86). The proof is complete.

Proof of Theorem 1.5. The claim (1.17a) is a direct consequence of Proposition 3.14. We prove the claim (1.17b), applying (3.5) and parabolic estimates to the function $E=$ $W(y, s)-a_{n_{0}}(s) \psi_{n_{0}}(y)$, where $W(y, s)=y^{\gamma}(\Phi-\pi / 2), a_{n_{0}}(s)=\left\langle W(\cdot, s), \psi_{n_{0}}\right\rangle$. A direct computation shows that $E$ solves an $m$-dimensional parabolic equation

$$
\begin{aligned}
E_{s} & =\Delta_{\zeta} E-\frac{\zeta \cdot \nabla_{\zeta} E}{2}+\frac{\gamma}{2} E+G, \\
G & :=g_{1}(y, W)-\left\langle g_{1}(\cdot, W(\cdot, s)), \psi_{n_{0}}\right\rangle \psi_{n_{0}}(y),
\end{aligned}
$$

where $\zeta$ denotes space variable in $\mathbb{R}^{m}, m=d-2 \gamma \in \mathbb{N}$, such that $y=|\zeta|$ and

$$
g_{1}(y, W)=g(W)=\frac{\Lambda_{k}}{2} y^{\gamma-2}\left[\sin \left(2 y^{-\gamma} W\right)-2 y^{-\gamma} W\right] .
$$

Due to (3.5), for every $L, M>0$ there is a constant $C=C(L, M)>0$ such that

$$
|E(\zeta, s)|+|G(\zeta, s)| \leq C \varepsilon(s)^{\gamma+2 \theta} \quad\left(L / 2<|\zeta|<2 M, s_{0}+1<s\right) .
$$

A similar argument to (and even simpler than) the proof of Proposition 3.14 shows

$$
\sum_{j=0}^{2} \sum_{i_{1}+\cdots+i_{m}=j}\left|\frac{\partial^{j} E}{\partial \zeta_{1}^{i_{1}} \cdots \partial \zeta_{m}^{i_{m}}}(\zeta, s)\right| \leq C^{\prime} \varepsilon(s)^{\gamma+2 \theta} \quad\left(L \leq|\zeta| \leq M, s_{0}+1<s\right) .
$$

Writing this estimate with self-similar variables, we obtain

$$
\sum_{j=0}^{2}\left|\frac{\partial^{j} \Phi}{\partial y^{j}}(y, s)-a_{n_{0}}(s) \phi_{n_{0}}^{(j)}(y)\right| \leq C^{\prime} \varepsilon(s)^{\gamma+2 \theta} \quad\left(L \leq y \leq M, s_{0}+1<s\right),
$$

whence the result $(1.17 \mathrm{~b})$.

The claim (ii) follows from (i) and identity (1.5), i.e., $|\nabla F|^{2}=\left(u_{r}\right)^{2}+\Lambda_{k} r^{-2} \sin ^{2} u$. Indeed, for $r \leq K \varepsilon(s) \sqrt{T-t}$, there holds

$$
\begin{aligned}
\left|\left(u_{r}\right)^{2}-\left(u_{1, r}\right)^{2}\right| & \leq 2\left|u_{1, r}\right| \cdot \frac{\varepsilon_{0}(s)^{2 \theta}}{\varepsilon(s) \sqrt{T-t}} \leq \frac{C \varepsilon_{0}(s)^{2 \theta}}{\varepsilon(s)^{2}(T-t)} \\
\frac{\Lambda_{k}}{r^{2}}\left|\sin ^{2} u-\sin ^{2} u_{1}\right| & \leq \frac{\Lambda_{k}}{r^{2}} \cdot 2\left|u_{1}(r, t)\right|\left|u-u_{1}\right| \\
& \leq \frac{\Lambda_{k}}{r^{2}} \cdot 3\left(\frac{r}{\varepsilon(s) \sqrt{T-t}}\right)^{k} \varepsilon(s)^{2 \theta} H\left(\frac{r}{\varepsilon(s) \sqrt{T-t}}\right) \leq \frac{C \varepsilon(s)^{2 \theta}}{\varepsilon(s)^{2}(T-t)}
\end{aligned}
$$


where $u_{1}=u_{1}(r, t):=U_{1}(r / \varepsilon(s) \sqrt{T-t})$. When $L \leq r / \sqrt{T-t} \leq M$, we have

$$
\begin{aligned}
\left|\left(u_{r}\right)^{2}-\left(\tilde{u}_{r}^{*}\right)^{2}\right| & \leq C(L, M) \varepsilon_{0}(s)^{\gamma}(T-t)^{-1 / 2} \cdot\left|a_{n_{0}}^{*}(s)\right| \frac{\varepsilon(s)^{\gamma}}{\sqrt{T-t}} \leq \frac{C(L, M) \varepsilon(s)^{2 \gamma}}{T-t}, \\
\frac{\Lambda_{k}}{r^{2}}\left|\sin ^{2} u-\sin ^{2} \tilde{u}^{*}\right| & \leq \frac{2 \Lambda_{k}}{r^{2}}\left(\left|\cos \tilde{u}^{*}\right|\left|u-\tilde{u}^{*}\right|+\frac{1}{2 !}\left|u-\tilde{u}^{*}\right|^{2}\right) \leq \frac{C(L, M)}{T-t} \varepsilon(s)^{2 \gamma+2 \theta},
\end{aligned}
$$

where $\tilde{u}^{*}=\tilde{u}^{*}(r, t):=u^{*}(r / \sqrt{T-t}, t)$. Therefore the desired estimates follow from (1.5). The proof is now complete.

Acknowledgement. The second author was partly supported by Grant-in-Aid for scientific research (18K03373). This work was (partly) supported by Osaka City University Advanced Mathematical Institute (MEXT Joint Usage/Research Center on Mathematics and Theoretical Physics).

\section{References}

[1] R. Adams and J. Fournier, Sobolev spaces, Second edition, Pure and Applied Mathematics, Elsevier/Academic Press, Amsterdam, 2003.

[2] K. Anada and T. Ishiwata, Blow-up rates of solutions of initial-boundary value problems for a quasilinear parabolic equation, J. Differential Equations 262 (2017), 181-271.

[3] S. B. Angenent, J. Hulshof, and H. Matano, The radius of vanishing bubbles in equivariant harmonic map flow from $D^{2}$ to $S^{2}$, SIAM J. Math. Anal. 41 (2009), 1121-1137.

[4] P. Biernat, Non-self-similar blow-up in the heat flow for harmonic maps in higher dimensions, Nonlinearity 28 (2015), 167-185.

[5] P. Biernat and P. Bizoń, Shrinkers, expanders, and the unique continuation beyond generic blowup in the heat flow for harmonic maps between spheres, Nonlinearity 24 (2011), 2211-2228.

[6] P. Biernat and Donninger R., Construction of a spectrally stable self-similar blowup solution to the supercritical corotational harmonic map heat flow, Nonlinearity 31 (2018), 3543-3566.

[7] P. Biernat, Donninger R., and B. Schörkhuber, Stable self-similar blowup in the supercritical heat flow of harmonic maps, Calc. Var. Partial Differential Equations 56 (2017), 31pp.

[8] P. Biernat and Y. Seki, Type II blow-up mechanism for supercritical harmonic map heat flow, Int. Math. Res. Not. (IMRN) 23 (2019), 407-456.

[9] P. Bizoń and A. Wasserman, Nonexistence of shrinkers for the harmonic map flow in higher dimensions, Int. Math. Res. Not. (IMRN) 17 (2015), 7757-7762.

[10] Y. M. Chen and M. Struwe, Existence and partial regularity results for the heat flow for harmonic maps, Math. Z. 201 (1989), 83-103.

[11] J. Eells and A. Ratto, Harmonic Maps and Minimal Immersions with Symmetries: Methods of Ordinary Differential Equations Applied to Elliptic Variational Problems, Annals of Mathematics Studies, vol. 130, Princeton University Press, Princeton, 1993.

[12] H. Fan, Existence of the self-similar solutions in the heat flow of harmonic maps, Science in China Series A: Mathematics 42 (1999), 113-132.

[13] S. Filippas, M. A. Herrero, and J. J. L. Velázquez, Fast blow-up mechanisms for sign-changing solutions of a semilinear parabolic equation with critical nonlinearity, R. Soc. Lond. Proc. Ser. A. 456 (2000), 2957-2982. 
[14] A. Gastel, Singularities of first kind in the harmonic map and Yang-Mills heat flows, Math. Z. 242 (2002), 47-62.

[15] P. Germain, T.-E. Ghoul, and Miura H., On Uniqueness for the Harmonic Map Heat Flow in Supercritical Dimensions, Comm. Pure Appl. Math. (2017), 2247-2299.

[16] P. Germain and M. Rupflin, Selfsimilar expanders of the harmonic map heat flow, Ann. Inst. Henri Poincare (C) Non Linear Analysis (2011), 743-773.

[17] T.-E. Ghoul, S. Ibrahim, and V. T. Nguyen, On the stability of type II blowup for the 1-corotational energy-supercritical harmonic heat flow, Anal. PDE 12 (2019), 113-187.

[18] J.-S. Guo, H. Matano, and C.-C. Wu, An application of braid group theory to the finite time dead-core rate, J. Evol. Equ. 10 (2010), 835-855.

[19] J.-S. Guo and C.-C. Wu, Finite time dead-core rate for the heat equation with a strong absorption, Tohoku Math. J. (2) 60 (2008), 37-70.

[20] J. Harada, Non self-similar blow-up solutions to the heat equation with nonlinear boundary conditions, Nonlinear Anal. 102 (2014), 36-83.

[21] _ Construction of type II blow-up solutions for a semilinear parabolic system with higher dimension, Calc. Var. Partial Differential Equations 56 (2017), 36pp.

[22] M. A. Herrero and J. J. L. Velázquez, Explosion de solutions d'équations paraboliques semilinéaires supercritiques, C. R. Acad. Sci. Paris Sér. I Math. 319 (1994), 141-145.

[23] _ _ A blow up result for semilinear heat equations in the supercritical case, unpublished.

[24] _ On the melting of ice balls, SIAM J. Math. Anal. 28 (1997), 1-32.

[25] _ Singularity patterns in a chemotaxis model, Math. Ann. 306 (1996), 583-623.

[26] W. Jäger and H. Kaul, Rotationally symmetric harmonic maps from a ball into a sphere and the regularity problem for weak solutions of elliptic systems, J. Reine Angew. Math. 343 (1983), 146-161.

[27] H. Matano, Blow-up in nonlinear heat equations with supercritical power nonlinearity, Contemp. Math., vol. 446, Amer. Math. Soc., Providence, RI, 2007, pp. 385-412.

[28] N. Mizoguchi, Blow-up rate of type II and the braid group theory, Trans. Amer. Math. Soc. 363 (2011), 1419-1443.

[29] P. Raphaël and R. Schweyer, Stable blowup dynamics for the 1-corotational energy critical harmonic heat flow, Comm. Pure Appl. Math. 66 (2013), 414-480.

[30] _ Quantized slow blow-up dynamics for the corotational energy-critical harmonic heat flow, Analysis \& PDE 7 (2014), 1713-1805.

[31] Y. Seki, On exact dead-core rates for a semilinear heat equation with strong absorption, Comm. Contemp. Math. 13 (2011), 1-52.

[32] _ . Type II blow-up mechanisms in a semilinear heat equation with critical Joseph-Lundgren exponent, J. Funct. Anal. 275 (2018), 3380-3456.

[33] _ Refined estimates on blow-up solutions of $k$-equivariant harmonic map heat flow, in preparation.

[34] Y. Seki, Y. Sugiyama, and J. J. L. Velázquez, Multiple peak aggregations for the Keller-Segel system, Nonlinearity 26 (2013), 319-352.

[35] T. Senba, A fast blowup solution to an elliptic-parabolic system related to chemotaxis, Adv. Differential Equations 11 (2006), 981-1030.

[36] M. Struwe, Geometric evolution problems, Nonlinear Partial Differential Equations in Differential Geometry, AMS/IAS/Park City Mathematics Institute, Houston, 1996, pp. 259-339.

[37] J. B. van den Berg, J. Hulshof, and J. King, Formal asymptotics of bubbling in the harmonic map heat flow, SIAM J. Appl. Math. 63 (2003), 1682-1717. 